\title{
1 AAV2/9-mediated gene transfer into murine lacrimal gland leads to a long-term 2 targeted tear film modification
}

3 Benoit Gautier ${ }^{1, *}$, Lena Meneux ${ }^{1}$, Nadège Feret ${ }^{1}$, Christine Audrain ${ }^{2}$, Laetitia Hudecek ${ }^{1,3}$, Alison

4 Kuony $^{1,4}$, Audrey Bourdon ${ }^{5}$, Caroline Le Guiner ${ }^{5}$, Véronique Blouin ${ }^{5}$, Cécile Delettre ${ }^{1}$ and Frédéric 5 Michon $^{1, *}$

6

$7 \quad{ }^{1}$ Institute for Neurosciences of Montpellier, Univ Montpellier, INSERM, Montpellier, France

$8 \quad 2$ TarGeT, Nantes University, INSERM UMR 1089, CHU Nantes, Nantes, France

$9 \quad{ }^{3} \mathrm{MRI}$, Biocampus, Univ Montpellier, CNRS, INSERM, Montpellier, France

$10{ }^{4}$ Cell Adhesion and Mechanics Lab, Université de Paris, CNRS, Institut Jacques Monod, Paris,

11 France

$12{ }^{5}$ INSERM UMR 1089, Université de Nantes, CHU de Nantes, Nantes, France

$14{ }^{*}$ Co-corresponding authors

15 Emails: benoit.gautier@inserm.fr, frederic.michon@inserm.fr

16

17 Keywords: AAV, lacrimal gland, tear film, cornea, NGF 


\section{Abstract}

19 Corneal blindness is the fourth leading cause of blindness worldwide. Since the corneal epithelium 20 is constantly renewed, non-integrative gene transfer cannot be used to treat corneal diseases. In 21 numerous of these diseases, the tear film has been reported to be defective. Tears are a complex 22 biological fluid secreted by the lacrimal apparatus. Their composition is modulated according to the 23 context. For instance, after a corneal wound, the lacrimal gland secretes reflex tears, which contain 24 specific growth factors supporting the wound healing process. In specific pathological contexts, 25 such as dry eye diseases, the tear composition can support neither corneal homeostasis, nor 26 wound healing. Here, we propose to use the lacrimal gland as bioreactor to produce and secrete 27 specific factors to support corneal physiology. In this study, we used an AAV2/9-mediated gene 28 transfer to supplement the tear film. First, we demonstrate that a single injection of AAV2/9 is 29 sufficient to transduce all epithelial cell types of the lacrimal gland efficiently and widely. Then, we 30 show that lacrimal gland physiology and corneal integrity are maintained after the injection of an 31 AAV2/9-mediated nerve growth factor expression in the lacrimal gland. Remarkably, this injection 32 induces an important and long-lasting secretion of this growth factor in the tear film. Altogether, our 33 findings provide a new clinically applicable approach to tackle corneal blindness. 


\section{Introduction}

To ensure clear vision, the anterior parts of the eye, namely the cornea and lens, need to be fully transparent. While the lens is protected by being located inside the eye, the cornea is the most external tissue of the eye, and thus prone to environmental aggressions. This ectodermal organ is subjected to life-long cell renewal, which relies on stem and progenitor cells ${ }^{1}$. To coordinate epithelium homeostasis, corneal microenvironment is composed of epithelial cell-cell communication, dense innervation, and tear film. The latter is the source of corneal hydration and nutrients for the epithelium². Moreover, after wounding, the tear composition changes to support corneal wound healing, through a modification of the factors secreted by the lacrimal gland (LG) ${ }^{3}$. The tear composition change requires an efficient sensory network of the cornea. The dense corneal innervation is essential to maintain corneal physiology. First, blinking and tear composition adaptation depends on the activity of corneal sensory nerves. Furthermore, the neurotrophic factors released by the nerves for the epithelium are crucial for homeostasis and wound healing ${ }^{4}$. Among the most prominent causes for corneal defects, three are making most of the influx of patients in hospital. First cause, physical wounds, such as abrasions ${ }^{5}$, are mostly caused by small foreign objects that scratch the epithelium. This injury is painful, and the subsequent oedema provokes photophobia and impairs visual acuity. If the particle gets embedded within the epithelium, corneal irregularities might form, resulting in continuous pain and significant visual disruption ${ }^{6}$. Dry eye diseases account for the second cause of corneal defects. Dry eye diseases can arise from genetic disease, such as Gougerot-Sjörgren syndrome ${ }^{7}$, or from ageing, as up to $1 / 3$ of the elder population can be affected ${ }^{8,9}$. The altered tear film has an imbalanced composition, offering less nutrients and growth factors to the corneal epithelium, which in turn affects corneal homeostasis. Consequently, persistent epithelial defects appear, such as ulceration, melting and perforation, impairing sight ${ }^{10}$. Third cause, neurotrophic keratitis is due to a partial or complete loss of corneal innervation, causing a defected corneal homeostasis ${ }^{11}$. Neurotrophic keratitis results from defected corneal wound healing after abrasion or transplant, from neurodegenerative diseases, or from chronic metabolic diseases, such as diabetes. Currently 415 million adults globally are diagnosed with diabetes, and the World Health Organization projected that there will 
be 640 million adults by $2040^{12}$. While being underdiagnosed, diabetic keratopathy affects 47 to $64 \%$ of diabetic adults ${ }^{13}$. The main symptom of neurotrophic keratitis is corneal ulceration and perforation.

The current treatments for these corneal defects are topical and consist in eye drops, which can be supplemented with autologous serum ${ }^{14}$, or nerve growth factor (NGF) in the case of neurotrophic keratitis $^{15}$. Not only these treatments are heavy for patients, especially autologous serum, but the frequent lack of patient compliance to a prescribed eyedrop regimen lead to increased sight defects $^{16}$.

These treatments solely rely on the addition of an external eye drop solution mimicking the tear film composition without considering the LG which produces, secretes, and modulates the tear film composition. Consequently, using LG directly as a bioreactor would constitute an appealing alternative strategy to modulate the tear film composition. This could be achieved by adenovirusassociated virus (AAV) vector-mediated gene transfer into LG. Indeed, AAV vectors present many advantages for gene delivery. They efficiently transduce a broad range of cells in which they allow long-lasting transgene expression. Importantly, they trigger limited/mild immunogenic responses in vivo which overall asserts their biosafety ${ }^{17}$. Numerous AAV-based gene therapies have emerged as evidenced by many ongoing clinical trials, namely for neurodegenerative, neuromuscular, cardiovascular, ocular genetic diseases and cancer $^{18,19}$. Only three AAV-based gene therapies have been approved by the FDA, among which two are still used. Luxturna ${ }^{\circledR}$ (voretigene neparvovec) is used for the treatment of biallelic RPE65 mutation-associated retinal dystrophy ${ }^{20}$ while Zolgensma ${ }^{\circledR}$ (onasemnogene abeparvovec-xioi) is delivered to paediatric patients under two years of age suffering from spinal muscular atrophy ${ }^{21}$. Importantly, in the field of ophthalmology, the majority of studies using AAV-based gene delivery have focussed on retina and to a lesser extent on cornea, while very few data are available on AAV-based gene transfer into $\mathrm{LG}^{22-24}$. In this study, we present a novel strategy to modulate corneal physiology through targeted tear film modification by transferring a gene of interest into LG. First, we confirmed that AAV-mediated gene transfer was feasible in murine LG using AAV2/5 or AAV2/9. After demonstrating that all epithelial cell types are prone to AAV-mediated gene transfer, we used AAV2/5 and AAV2/9-mediated 
91 murine nerve growth factor (mNGF), as proof of concept, to establish the parameters for efficient

92 gene transfer, allowing a targeted modification of tear composition. We investigated the impact of

93 AAV serotype impact on secreted protein level and chose AAV2/9 vector to investigate the duration

94 of tear film modulation, as well as the safety of such an approach on the cornea. Altogether, our

95 results demonstrate that a single AAV2/9 injection into murine LG could be used to specifically

96 modify the tear film and consequently to support corneal physiology in pathological contexts, such

97 as neurotrophic keratitis, or recurring abscesses. 


\section{Results}

\section{AAV2/9 and AAV2/5 mediate an efficient gene transfer in the lacrimal gland}

To investigate the use of the LG as bioreactor for protein secretion in the tear film, we first established an injection protocol that allows an efficient AAV-mediated gene transfer after a single injection into murine LG. We used AAV2/9-CAG-GFP and AAV2/5-CAG-GFP to monitor the extent of vector diffusion within LG. The immunostaining of GFP demonstrated that these AAV serotypes induced GFP expression in all territories of the LG (Figure 1). To rule-out a possible tropism of AAV serotypes for a specific-cell type, we looked at GFP expression, one month after injection, in two different LG epithelial cell populations, namely the E-Cadherin (E-Cadh) positive cells, located in the acinar compartment, and the Keratin19 (Krt19) positive subpopulation, specifically localized in the ducts ${ }^{25}$. The GFP / E-Cadh co-labelling showed that almost all acinar cells were GFP positive regardless of the used serotype (Figure 2A, S1). Similarly, the GFP / Krt19 co-labelling demonstrated that most of the ductal cells were GFP positive after AAV2/9-CAG-GFP and AAV2/5CAG-GFP injection (Figure 2B, S2).

To evaluate a possible discrepancy between AAV serotypes 2/5 and 2/9 in the transduction efficiency, we analyzed by Western-blot the levels of GFP protein expressed in LG after injection of AAV2/9- or AAV2/5-CAG-GFP (Figure 3). As expected, while in the LG injected with AAV2/9 or AAV2/5 empty capsids, no GFP protein was detected (Figure 3A), whereas high levels of GFP were detected in the LG injected with AAV2/9 or AAV2/5-CAG-GFP respectively. Interestingly, when comparing the two AAV serotypes, GFP levels were found significantly higher after AAV2/9CAG-GFP injection than after AAV2/5-CAG-GFP injection (Figure 3B).

Taken together, our results demonstrate that LG can be widely transduced by AAV2/9 and AAV2/5, and that serotype 2/9 exhibits a higher transduction efficiency.

\section{The transgene expression leads to the secretion of the resulting protein in the tear film}

To evaluate if AAV2/9 and AAV2/5 serotypes have a different impact on the amount of protein secreted in the tear film, we chose to express the murine nerve growth factor (mNGF) in the LG, using either AAV2/9 (AAV2/9-CAG-mNGF) or AAV2/5 (AAV2/5-CAG-mNGF). Following AAV 
127 injection into LG, the transgene expression should lead to the secretion of mNGF in the tear film.

128 To assess this secretion, we measured mNGF level in the tear film, using ELISA and Western-blot 129 analysis (Figure 4). Tear film contains a basal level of $\mathrm{mNGF}^{26}$, which can have two forms ${ }^{27,28}$. The 130 pro-mNGF, of higher molecular weight is cleaved to generate the mature mNGF, the active form.

131 As expected from the GFP results, injection of AAV2/9 and AAV2/5 CAG-mNGF induced a 132 significant increase of the mNGF levels in the tear film, whereas only endogeneous basal 133 expression levels of mNGF were detected with the empty AAV vectors. Moreover, consistant with 134 the GFP expression data in LG, mNGF amount in the tear film, was more than 3-fold higher after 135 AAV2/9 injection than after AAV2/5 injection (Figure 4). Moreover, Western blot analysis confirmed 136 that injection of AAV2/9 and AAV2/5 CAG-mNGF led to a significant increase of the total mNGF 137 level in the tear film (Figure 5A and 5B). Importantly, this analysis revealed that mNGF was

138 consistently found in the tear film in its pro-mNGF form (Figure 5C). However, the analysis of the 139 mature mNGF showed a maturation process (Figure 5D). Indeed, the mature mNGF form was 140 close to absent from the tear film of mice injected in LG with empty AAV2/9 or AAV2/5, whereas a 141 large amount of mature mNGF was detected after AAV-mNGF gene transfer. Notably, AAV2/9-

142 CAG-mNGF gave rise to 4-times more of the mature form than with AAV2/5-CAG-mNGF injection 143 (Figure 5D).

AAV2/9-mediated gene transfer induces a dose-dependent and long-term protein secretion

146 Consequently to our results indicating a better protein secretion using AAV2/9 compared to 147 AAV2/5, we chose to focus on the serotype 2/9. To establish the optimal amount of vector 148 genomes $(\mathrm{vg})$ to be injected in LG, we tested three doses and checked the amount of secreted 149 mNGF one month after injection (Figure 6A). While injection of $10^{9} \mathrm{vg}$ did not lead to a drastic 150 increase of the mNGF secretion, a single injection of $10^{11} \mathrm{vg}$ induced the secretion of $75 \mathrm{ng}$ of $151 \mathrm{mNGF}$ per $\mathrm{mL}$ of tear film. This high concentration represents a 10-time increase in comparison to 152 physiological mNGF secretion during cornea wound healing process, where a concentration of 7.5 $153 \mathrm{ng} / \mathrm{mL}$ was found seven days after abrasion (Figure S3). We concluded that $10^{11} \mathrm{vg} / \mathrm{LG}$ was 154 suitable to have a significantly high increase of the protein secretion in the tear film. 
155 Subsequently, we evaluated the dynamics of the mNGF secretion over a 6-month period (Figure

156 6B). The injection of $10^{11} \mathrm{vg}$ into the $L G$ induced a significant increase of the mNGF level found in

157 the tear film already after a week, before reaching a peak at 30 days post injection. The mNGF

158 secretion then decreased to reach a plateau, 120 days after injection with stable expression during

159 the 60 following days. However, although injection of $10^{10} \mathrm{vg} / \mathrm{LG}$ of AAV2/9-CAG-mNGF or

160 AAV2/5-CAG-mNG induced a peak 30 days after injection, only the serotype 2/9 induced a

161 significant increase of the mNGF level in the tear film (Figure S4). Nonetheless, the secreted

162 mNGF level for both serotypes decreased and were no more different than the basal level. Taken

163 together, these results show the long-lasting protein secretion when injecting $10^{11} \mathrm{vg} / \mathrm{LG}$,

164 compared to $10^{9}$ or $10^{10} \mathrm{vg}$, and confirm that $A A V 2 / 9$ is more efficient than AAV2/5.

165

166 AAV2/9 vector genome copies in the murine LG correlates with the amount of protein 167 secreted in the tear film

168 To further analyze further the use of $10^{11} \mathrm{vg} / \mathrm{LG}$, we investigated the biodistribution of the

169 AAV2/9-CAG-mNGF vector after injection into LG. We measured the amount of vector genomes

170 (vg) per diploid genome (dg) in LG, liver and heart, one month after vector injection (Figure 7A). All

171 injected mice showed vector genome copies in LG, indicating that the injection technique is reliable

172 and reproducible. Interestingly, we found around $0.25 \mathrm{vg} / \mathrm{dg}$ in the LG, meaning that an average

173 of 1 out of 4 cells was transduced with 1 copy of the AAV2/9-CAG-mNGF vector, which reflects an

174 efficient gene transfer. Despite the detection of the AAV2/9-CAG-mNGF vector genome in liver and

175 heart of injected mice, the levels were very low to negligeable, with 14 and 80-times less $\mathrm{vg} / \mathrm{dg}$ in

176 liver and heart respectively than in LG.

177 Then, we compared the $\mathrm{vg} / \mathrm{dg}$ values to the concentration of $\mathrm{mNGF}$ in the tear film of the same

178 injected mice (Figure 7B). Remarkably, we found a nice correlation between the vg / dg values

179 detected in the LG and the amounts of secreted mNGF in the tears. The increase of $\mathrm{vg} / \mathrm{dg}$ value

180 corresponded to a higher mNGF secretion.

181 Taken together, these results confirm the high transduction efficiency displayed by AAV2/9, which

182 is additionally correlated with the amount of protein secreted in the tear film. Moreover, they 
showed a very limited distribution of AAV2/9 vector to peripheral organs after a single injection into

184 murine LG.

\section{AAV2/9-mediated mNGF secretion does not affect LG physiology nor corneal integrity}

187 After resolving the parameters for an efficient AAV2/9-mediated mNGF gene transfer, its 188 biodistribution, and induced mNGF secretion, we investigated the impact of the mNGF over 189 secretion on LG physiology and corneal integrity. We injected $10^{11} \mathrm{vg} / \mathrm{LG}$ of AAV2/9-CAG-mNGF 190 and measured the impact on tear volume and protein concentration in tears over a period of 6 191 months. (Figure 8A and 8B). Simultaneously to the increase of mNGF secretion (Figures 4-6), we 192 detected a significant increase of the tear volume from 7 to 120 days post-injection, when 193 compared to the injection of AAV2/9 empty capsid (Figure 8A). Interestingly, this increase of the 194 tear volume was matched with a stability of the protein concentration in tears (Figure 8B). 195 Moreover, to rule out any impact of the injection procedure on LG physiology, we analyzed the 196 tears before and after the AAV2/9 empty capsid injection (Figure S5). Importantly, after injection of

197 the AAV2/9 empty capsid and during the whole 6-month-period, no modification of tear volume nor 198 of total protein concentration was observed (Figure S5). This rule out any impact of the injection 199 procedure itself on LG physiology.

200 In addition, we used fluorescein staining to monitor the corneal epithelium integrity and visualize 201 any adverse effect that the injection procedure and the tear film modification could have on the 202 cornea. Fluorescein stains areas where the corneal epithelial barrier is defected ${ }^{1}$, as after corneal 203 abrasion (Figure $8 C$ ). During the 180 days after AAV2/9 injection, we never observed any 204 fluorescein staining, whether we injected an empty capsid, or induced mNGF over secretion. We 205 concluded that AAV2/9 injection does not impact the corneal epithelium integrity.

206 To investigate further the effect of the mNGF over secretion on cornea, we visualized corneal 207 innervation with $\beta$ III tubulin immunolabelling, which is a pan neuronal marker. We showed that the 208 gross morphology of corneal fibers was not affected by the over secretion of mNGF in the tear film 209 (Figure 9A). Furthermore, we performed von Frey tests on the corneas of injected mice. We 210 demonstrated that the constant over secretion of mNGF did not modify corneal sensitivity (Figure 
211 9B). All together, these results demonstrate that the injection procedure and mNGF over secretion

212 respect the LG physiology and the corneal integrity.

213 


\section{Discussion}

215 Among the sight threatening diseases, corneal defects are the fourth cause for blindness globally.

216 Except for physical harm, corneal blindness often results from a combination of intrinsic and 217 extrinsic causes. In the case of genetic diseases, such as aniridia ${ }^{29,30}$, or systemic diseases, such 218 as diabetes ${ }^{13}$, corneal defect is often associated with corneal microenvironment dysregulation ${ }^{31}$.

219 The aim of this study was to establish an innovative and attractive strategy to tackle corneal 220 defects by sustainably modulating the tear film composition. For this purpose, we chose an AAV221 mediated gene transfer to use LG as a bioreactor producing specific transgenes. Nevertheless, 222 several major variables are crucial to achieve an efficient transduction of the target cells, and thus 223 require to be well-designed ${ }^{17,32}$. First, the route of administration determines both the efficacy and 224 the biosafety pattern of an AAV-based gene transfer. In this study, we used a local injection into $225 \mathrm{LG}$ as this organ is easily accessible by surgery. Moreover, we showed that a local injection allows 226 concentration and time residence of AAV vectors in vicinity to the target cells, limits the 227 biodistribution of the vector to non-targeted tissues and thus the risk of toxicity. For instance, local 228 injection is well-established for ocular diseases, particularly in retinal disorders, as several 229 anatomical sites are commonly targeted: subretinal, intravitreal, intracameral, suprachoroidal and 230 topical $^{33,34}$. Furthermore, the injection method is a key parameter to reach a large diffusion in the 231 target organ. While a systemic injection leads to large diffusion in all the organism including the 232 target organ ${ }^{35}$, when using local injection, the development of specific injection procedures are 233 necessary. For example, several injections at precise coordinates are required for a large diffusion 234 in the brain $^{36}$. Interestingly, a pneumatic picopump system applying multiple short-time pressure 235 pulses has been reported to transduce the whole sciatic nerve of rodents ${ }^{37}$. In this study, we used 236 34-gauge beveled needle linked to a $10-\mu \mathrm{L}$ Hamilton syringe to perform injections into LG. We 237 injected $3 \mu \mathrm{l}$ of AAV vectors by following the path of the LG main duct. This procedure of injection 238 led to a large diffusion in the gland, and showed an efficient gene transfer in all epithelial cell types 239 in the murine LG.

240 The promoter used to drive the expression of the transgene is a second parameter determining the 241 transduction efficiency and the biosafety pattern of the injection ${ }^{17,32}$. Indeed, the promoter controls 
242 the transgene expression level. Commonly, AAV-mediated gene transfer uses an ubiquitous and 243 strong promoter, such as CAG and CMV, to achieve high transgene expression ${ }^{38,39}$. Moreover, 244 researchers usually employ such promoters when trying to establish a proof of concept for AAV245 mediated gene transfer into a specific organ. In this study, we utilized a CAG promoter to provide a 246 proof of concept of the tear fluid composition modulation after an AAV-mediated gene transfer into 247 murine LG. On the other hand, a high transgene expression level is not always desired. For 248 instance, transgene over expression above physiological levels has been reported to be toxic ${ }^{40}$. 249 This toxicity must be correlated to the serotype of the AAV vector. Indeed, the serotype represents 250 another parameter influencing both the transduction efficiency and the biosafety of an injection as 251 each AAV serotypes exhibit different cell and tissue tropisms ${ }^{41}$. Therefore, the combination of the 252 route of administration, the AAV serotype, the promoter driving the transgene expression and the 253 dose of vector have to be carefully designed as it may induce transgene expression in off-target 254 tissues and thus lead to dramatic toxicities ${ }^{42}$. In this study, we compared the AAV serotypes 2/5 255 and 2/9 to first transduce the murine LG efficiently and then to modulate the tear film composition. 256 Notably, while both serotypes led to an efficient gene transfer in the LG, AAV2/9 gave a better yield 257 of GFP production and mNGF secretion compared to AAV2/5. Interestingly, although the difference 258 of transduction efficiency was reported previously ${ }^{24}$, the 2 to 4 time fold in mNGF secretion is 259 startling. Indeed, until now, no study has reported a transgene secretion in the tear fluid after an 260 AAV-mediated gene transfer into LG. Furthermore, we tested increasing dose of AAV2/9 to 261 evaluate the mNGF secretion in the tear film. Interestingly, while $10^{11} \mathrm{vg} / \mathrm{LG}$ dose led to the 262 highest mNGF secretion, $10^{10} \mathrm{vg} / \mathrm{LG}$ was sufficient to induce a significant secretion. Given that 263 we used the serotype $2 / 9$, known to show high heart and liver tropism in rodent ${ }^{43}$, and a strong 264 CAG promoter, we performed a biodistribution study for AAV2/9. This study was paramount to 265 determine the potential off-targets of our strategy, which may lead to both unwanted toxicity and 266 immunogenicity. At the highest injected dose of $10^{11} \mathrm{vg} / \mathrm{LG}$, we showed an average of 0.25 $267 \mathrm{vg} / \mathrm{dg}$ in LG, only $0.017 \mathrm{vg} / \mathrm{dg}$ in liver and $0.003 \mathrm{vg} / \mathrm{dg}$ in heart of injected mice, which 268 corresponded to 14- and 80-times less vg / dg than in LG respectively. Importantly, these levels of 
AAV2/9 found in liver and heart were very low to negligible, from 100 to 10000 times lower than

270 those obtained after intravenous ${ }^{39}$ or intrathecal ${ }^{44}$ injections.

271 Different strategies exist to limit the transgene expression in off-targets and its related adverse 272 effects, including the use of cell-type specific promoters ${ }^{39}$ or the incorporation of miRNA binding 273 sites in the AAV gene expression cassette ${ }^{45}$. We can speculate that the use of a 10-time lower 274 dose of AAV2/9 vector, providing a high secretion of mNGF, could bring a lower off-target 275 transduction. Injection of $10^{10} \mathrm{vg} / \mathrm{LG}$ of AAV led to a faster secretion of mNGF with AAV2/9 276 compared to AAV2/5. After a peak 30-day post-injection, the mNGF secretion decreased to the 277 physiological level with both serotypes. However, injection of $10^{11} \mathrm{vg} / \mathrm{LG}$ results in over secretion 278 of mNGF during several months. This is an important aspect for the subsequent use of this 279 method. While a lower dose could be used for transitory pathologies, such as corneal graft, or 280 recurrent corneal abscesses, a higher dose could be of interest for chronical pathologies, such as 281 neurotrophic keratitis, or dry eye diseases.

282 The long-term secretion of mNGF is a remarkable discovery, which must be linked to the LG 283 physiology. As all epithelial organs, such as skin, cornea or mammary glands, the epithelial 284 compartment contains stem cells regenerating continuously the organ, and healing it if 285 necessary ${ }^{46}$. The AAV vector is a non-integrative vector that can be lost when cell division 286 occurs $^{47}$. Therefore, only non-proliferating cells will keep the transgene and lead to protein 287 secretion. Our results demonstrate that gene therapy is successful on this epithelial organ and 288 highlighting the low turn-over of epithelial cells in the LG. Knowing that 30 days after injection, 289 there was around one AAV2/9 vector genome in every 4 cells, it would be of great interest to 290 monitor over a long period of time to see which cells retain the AAV2/9 genome. This method 291 would give a deeper understanding of LG fundamental biology. Of course, this long term follow-up 292 could only be done at the cellular level, as with age, the LG physiology is randomly affected in 293 individuals $^{8}$. Therefore, measuring tear volume and tear protein content could be misleading 294 because of aging impact more than AAV2/9 injection. Nevertheless, we demonstrated that up to 6 295 months after injection into the murine LG, our approach has no detrimental impact on the LG 296 physiology. 
297 The presence of mature mNGF in the tear film is important for its functionality. We showed that in

298 the absence of mNGF over secretion, only a negligible part of the pro-mNGF was processed into

299 its mature form. Interestingly, the mNGF over secretion led to the presence of mature mNGF in the

300 tears. However, we cannot explain why after AAV2/9 injection, $57 \%$ of the total mNGF is matured,

301 and only $34 \%$ after AAV2/5 injection. We can only hypothesize a threshold effect leading to a

302 better pro-mNGF processing into mature mNGF when there is an increase of secretion in the tear

303 film.

304 Despite the large amount of mNGF and its constant presence on the cornea, we did not detect any 305 impact on corneal innervation, nor on corneal sensitivity. We hypothesize that the robustness of 306 corneal innervation maintains the system under control in physiological conditions, and mNGF 307 alone is not sufficient to disturb this system. Most likely, under pathological conditions, i.e., physical 308 harm, or neurotrophic keratopathy, the system would be sufficiently perturbed to visualize the 309 effect of mNGF on corneal innervation.

310 Collectively, our results show that LG gene therapy could be established to modify specifically the 311 tear film to support corneal physiology. The main challenge in using AAV vectors for epithelial 312 organ gene therapy is the high renewal rate of epithelial cells. Here, we demonstrate that there is a 313 non-renewing cell population in the LG that retains the secretory capabilities to produce a large 314 amount of mNGF for over 6 months. The long term over secretion of mNGF could replace the use 315 of NGF supplemented eyedrops, used to treat neurotrophic keratitis, such as observed in 316 diabetes $^{12}$ or neurodegenerative diseases ${ }^{48}$. Notably, by substituting mNGF with another gene, 317 other corneal defects could be treated. 


\section{Material and Methods}

\section{Study design}

321 The goal of this study was to assess the transduction pattern of AAV vector serotypes 2/5 and 2/9

322 after a single injection into murine LG. Then, to evaluate the efficiency and the safety of a AAV2/9-

323 mediated gene transfer of mNGF in the murine LG, for its secretion in the tear film. The main

324 readouts of this study included: the transduction pattern analyzed by $\mathrm{IHC}$ and Western blot, the

325 secretion of mNGF measured in tears by ELISA and Western blot, the AAV2/9 biodistribution by

326 qPCR, the injection biosafety by analyzing the corneal integrity and innervation, the volume of

327 tears and the protein concentration in tears. Experimental groups were sized according to the

328 literature to allow statistical analysis. No outliers were excluded from the study, except mice

329 exhibiting spontaneous eye damages after the surgery or during the experiments. Behavioral data

330 obtained from animals displaying eye damages unrelated to the abrasion procedure during the

331 study were excluded. Scientists who performed the experiments and analysis were blinded to the

332 group's identity. Data were analyzed by those carrying out the experiments and verified by the

333 supervisor.

334

\section{Cloning and vector production}

336 The recombinant AAV vectors developed were called AAV hereafter. Cloning of the enhanced GFP

337 (GFP) and the mNGF in pAAV and AAV vector productions were provided by the vector core of the

338 TarGeT (Translational Gene Therapy) Laboratory of Nantes, INSERM UMR 1089 (Nantes

339 University, France). Briefly, single-stranded AAV2/5 and AAV2/9 CAG-GFP, single-stranded

340 AAV2/5 and AAV2/9 CAG-mNGF vectors were obtained from pAAV CAG-GFP and pAAV CAG-

341 mNGF plasmids respectively, containing AAV2 inverted terminal sequences, CAG promoter and

342 BGH polyA signal. AAV2/5 and AAV2/9 CAG-GFP vectors were used to assess the transduction

343 pattern after injection in the murine LG. AAV2/5 and AAV2/9 CAG-mNGF vectors were used to

344 evaluate the efficiency of an AAV-based gene transfer in the LG allowing mNGF secretion in the

345 tear fluid. Single-stranded vectors containing AAV2/5 and AAV2/9 empty capsid served as 346 controls. 
347 Vector production was performed following the protocol of vector core of the TarGeT Laboratory of 348 Nates $^{49}$. Briefly, recombinant AAVs were manufactured by co-transfection of HEK293 cells and 349 purified by cesium chloride density gradients followed by extensive dialysis against phosphate350 buffered saline (PBS). Vector titers were determined by qPCR and expressed as vector genome 351 (vg) / $\mathrm{ml}$. The target amplicons correspond to the inverted terminal repeat (ITR) sequences, ITR-2 352 (ITR-2 Forward: GGAACCCCTAGTGATGGAGTT, ITR-2 Reverse: CGGCCTCAGTGAGCGA, 353 Taqman Probe used for vector titer: FAM- CACTCCCTCTCTGCGCGCTCG-BBQ).

\section{Animals included in this study}

356 All mice experiments were approved by the local ethical committee and the "ministère de la 357 recherche et de l'enseignement supérieur" (authorization 2016080510211993 version2). All the 358 procedures were performed in accordance with the French regulation for the animal procedure 359 (French decree 2013-118) and with specific European Union guidelines for the protection of animal 360 welfare (Directive 2010/63/EU). Mice were maintained on a $12 \mathrm{~h}$ dark, $12 \mathrm{~h}$ light cycle with a 361 humidity between 40 and $60 \%$ and an ambient temperature of $21-22{ }^{\circ} \mathrm{C}$.

362

\section{Surgery and Vector delivery}

364 Twelve-week-old Swiss/CD1 female mice (Janvier Labs, France) were injected into the right LG. 365 The LG injection of AAV vectors was performed under anesthesia with a mixture of Ketamine (70 $366 \mathrm{mg} / \mathrm{kg}$, Imalgene® 1000, Centravet, France) and Medetomidine (1 mg / kg, Domitor $\AA^{\circledR}$, Centravet, 367 France). One drop of Ocry-gel (Centravet, France) was applied to each eye. The skin on the cheek 368 under the right ear was disinfected with vetedine solution (Centravet, France) and ethanol $70 \%$ and 369 then cut above the LG location. Next, the viral solution was injected using a 34-gauge beveled 370 needle (Hamilton, reference 207434, Reno, NV, USA) linked to a 10- $\mu$ l Hamilton syringe (1701 RN 371 serie, Hamilton, reference 7653-01, Reno, NV, USA). Wound were closed by suture wires 372 (Novosyn $\AA$ 6/0, reference C0068006, B Braun) and then disinfected with vetedine solution 373 (Centravet, France). After surgery, mice were treated with Buprenorphine $(100 \mu \mathrm{g} / \mathrm{kg}$, 
374 Brupecare $\AA$, Centravet, France) and were woken up with Atipamezole (1 mg / kg, Antisedan®,

375 Centravet, France).

376 AAV vector solutions were prepared by diluting vectors at the right titer with sterile PBS and $0.01 \%$ 377 of Fast Green (Sigma-Aldrich, reference F7252, France). For all experimental studies, mice were 378 unilaterally injected in the right LG with $3 \mu \mathrm{l}$ of vectors indicated below. For the transduction pattern 379 study, AAV2/5 and AAV2/9 CAG GFP were injected at $10^{10} \mathrm{vg} / \mathrm{LG}$. For the dose response study, 380 AAV2/9 CAG-mNGF was injected at $10^{9}, 10^{10}$ or $10^{11} \mathrm{vg} / \mathrm{LG}$. For the kinetic study, AAV2/5 CAG$381 \mathrm{mNGF}$ was injected at $10^{10} \mathrm{vg} / \mathrm{LG}$ and AAV2/9 CAG-mNGF was injected at $10^{10}$ and $10^{11} \mathrm{vg} / \mathrm{LG}$.

382 For the biodistribution and the biosafety studies, AAV2/9 CAG-mNGF was injected at $10^{11} \mathrm{vg} / \mathrm{LG}$. 383 Animals injected with AAV2/5 or AAV2/9 empty capsid served as control.

\section{Corneal abrasion}

386 Corneal abrasions were performed as previously described ${ }^{1,3,50}$. Briefly, an ocular burr (Algerbrush 387 II, reference BR2-5 0,5 mm, Alger company) was used on mice that were anesthetized with a 388 mixture of Ketamine (70 mg/ kg, Imalgene® 1000 , Centravet, France) and Medetomidine (1 $389 \mathrm{mg} / \mathrm{kg}$, Domitor ${ }^{\circledR}$, Centravet, France). Abrasions were performed unilaterally. A fluorescein 390 solution (1\% in PBS, Sigma-Aldrich) was used to visualize the wound under a cobalt blue light. 391 After abrasion, one drop of Ocry-gel (Centravet, France) was applied to each eye, mice were 392 treated with Buprenorphine $\left(100 \mu \mathrm{g} / \mathrm{kg}\right.$, Brupecare ${ }^{\circledR}$, Centravet, France) and were woken up with 393 Atipamezole (1 mg / kg, Antisedan®, Centravet, France).

\section{Tissue collection and processing}

396 Tears were collected using a $1 \mu$ l-microcapillary (Sigma-Aldrich, reference P1424, France) for one 397 minute, 1 day before injection and 30 days post injection for the dose response and biodistribution 398 studies; 1 day before injection and 7, 30,60, 120 and 180 days post injection for the kinetic and 399 biosafety studies; 1 day before abrasion and 1, 3 and 7 days post abrasion for the corneal abrasion 400 study. 
401 For the transduction pattern and the biodistribution studies, mice were euthanized 30 days post

402 injection using pentobarbital $(54.7 \mathrm{mg} / \mathrm{mL}, 140 \mathrm{mg} / \mathrm{kg}$, Centravet, France). They were transcardially

403 perfused with sterile PBS and tissues were quickly dissected. Tissues were then fixed for $45 \mathrm{~min}$ in 404 4\% paraformaldehyde solution (AntigenFix, Diapath, reference P0014, France) at room 405 temperature or directly snap-frozen in liquid nitrogen and stored at $-80^{\circ} \mathrm{C}$ for $\mathrm{IHC}$ and 406 molecular/biochemical analysis respectively.

407 For whole cornea imaging, mice were euthanized by cervical dislocation, enucleated with curved 408 scissors by cutting the optic nerve. Collected eyes were then fixed for $20 \min$ in $4 \%$ 409 paraformaldehyde solution (AntigenFix, Diapath, reference P0014, France) at room temperature. 410 After PBS washes, eyes were dehydrated during $2 \mathrm{~h}$ in $50 \%$ ethanol/PBS and then stored at $4^{\circ} \mathrm{C}$ in $41170 \%$ ethanol/PBS.

\section{Tear volume, protein concentration and mNGF analyses in tears}

414 The volume of tears per minute $(\mu \mathrm{l} / \mathrm{min})$ and the protein concentration in tears $(\mathrm{mg} / \mathrm{ml})$ using the 415 BCA protein assay kit (Fisher Scientific, reference 10678484, France) were measured and 416 expressed as the mean \pm SD.

417 The mNGF level in tears upon a AAV2/9 CAG-mNGF injection in the LG was dosed using the 418 mNGF ELISA kit (Sigma-Aldrich, reference RAB1119, France) following the manufacturer's 419 recommendations. Measures were performed on a CLARIOstar microplate reader (BMG Labtech, 420 France), and analyzed using the CLARIOstar software (version $5.60 \mathrm{R} 2$ ). Results are expressed as 421 the mean \pm SD.

\section{IHC study}

424 The following antibodies were used for IHC studies: chicken anti-GFP (Aves Labs, reference GFP$4251020,1 / 1000$ ), mouse anti-Ecadh (BD Biosciences, reference 610182, 1/300), rabbit anti-Krt19 426 (Abcam, reference ab52625, 1/200), rabbit anti- $\beta$ III tubulin (Abcam, reference ab18207, 1/1000), 427 goat anti-chicken Alexa Fluor 488 (Thermo Fisher Scientific, reference A-32931, 1/500), goat anti428 mouse Alexa Fluor 568 (Thermo Fisher Scientific, reference A-11004, 1/500) and goat anti-rabbit 
Alexa Fluor 568 (Abcam, reference ab175471, 1/500), goat anti-rabbit Alexa Fluor 488 (Abcam,

430 reference ab11008, 1/500) and goat anti-rabbit Alexa Fluor 568 (Abcam, reference ab175471,

431 1/500). Nuclei were counterstained with Hoechst 33342 (Thermo Fisher Scientific, reference

432 H3570, 1/2000) and BioTracker NIR694 (Merck, reference SCT118, 1/400).

\section{Immunohistochemistry on LG frozen sections}

435 Following fixation, LG were incubated $24 \mathrm{~h}$ in two successive baths of $6 \%$ and $30 \%$ sucrose and 436 then embedded in Optimal Cutting Temperature (OCT tissue freezing medium, MM France, 437 reference F/TFM-C) and stored at $-80^{\circ} \mathrm{C}$. Longitudinal sections (10 $\mu \mathrm{m}$ of thickness) were cut 438 using a cryostat apparatus (LEICA CM3050). For primary antibodies produced in rabbit and 439 chicken, cryosections were blocked with a mixture of $5 \%$ Goat Serum (GS, Thermo Fisher 440 Scientific, reference 16210064), 5 \% of fish skin gelatin (FSG, Sigma-Aldrich, reference G7765) 441 and $0.1 \%$ triton $\mathrm{X}-100$ in PBS for $1 \mathrm{~h}$ at room temperature. For primary antibodies produced in 442 mouse (mouse anti-Ecadh), the blocking step described above was followed by an incubation with 443 goat anti-mouse immunoglobulins (Abcam, reference ab6668, 1/200) during $1 \mathrm{~h}$ at room 444 temperature. Cryosections were then incubated overnight at $4{ }^{\circ} \mathrm{C}$ with primary antibodies diluted in 445 GS/FSG/Triton/PBS mixture, washed three times with $0.1 \%$ triton X-100 / PBS and subsequently 446 incubated $1 \mathrm{~h}$ at room temperature with secondary antibodies diluted in GS / FSG / Triton / PBS 447 mixture. After several PBS washes, cryosections were mounted in Fluoromount-G mounting 448 medium (Invitrogen, reference 00-4958-02).

\section{Immunohistochemistry on whole cornea}

451 Eyes were rehydrated in $50 \%$ ethanol/PBS during $2 \mathrm{~h}$ and washed twice in PBS for 15 minutes at 452 room temperature. Corneas were dissected and permeabilized with $0.5 \%$ Triton-X-100/PBS on a 453 rocking agitator for $1 \mathrm{~h}$ and then blocked in $5 \%$ GS (Thermo Fisher Scientific, reference 454 16210064) $2.5 \%$ FSG (Sigma-Aldrich, reference G7765) in $0.1 \%$ Triton X-100/PBS at room 455 temperature. Corneas were incubated in primary antibody diluted in blocking solution overnight at $45644^{\circ} \mathrm{C}$ on a rocking agitator and rinsed in $0.1 \%$ Triton X-100/PBS at room temperature (three times 
$4571 \mathrm{~h}$ ). Next, samples were incubated with secondary antibodies as previously mentioned. After the 458 washes, nuclei were stained 10 minutes with BioTracker NIR694 (Merck, reference SCT118) and

459 washed in PBS. Corneas were cut at four corners and mounted in Fluoromount-G mounting 460 medium (Invitrogen, reference 00-4958-02), epithelium facing the coverslip.

\section{Imaging}

463 LG images were acquired using Zen Black software (version 2.3 SP1, Zeiss, France) on a LSM 464880 confocal microscope (Zeiss, France). Whole LG section images were obtained using a $46520 x$ / 0.8 objective while co-immunostaining of GFP with E-cadherin or Keratin19 proteins were 466 observed via $0.36 \mu \mathrm{m}$ step size z-stacks using a $63 x / 1.4$ oil immersion objective. Images were 467 then processed with Zen Black software (version 2.3 SP1, Zeiss, France) and Zen Blue lite 468 software (version 3.2, Zeiss, France).

469 Whole cornea images were acquired using the navigator module on a Leica Thunder Imager 470 Tissue microscope with Large Volume Computational Clearing (LVCC) process. Images were 471 obtained using a 20x/0.55 objective with LAS X software (3.7.4) and processed with Imaris 472 Bitplane software (version 9.8.0).

\section{Western blot}

475 Frozen LG were crushed with a pestle and mortar pre cooled at $-80^{\circ} \mathrm{C}$, solubilized in Pierce ${ }^{\circledR}$ RIPA 476 lysis buffer (Thermo Fisher Scientific, reference 89900, France) supplemented with protease 477 inhibitors (Halt ${ }^{\mathrm{TM}}$ Protease Inhibitor Cocktail, Thermo Fisher Scientific, reference 87786, France), 478 homogenized on a rotating wheel at $4^{\circ} \mathrm{C}$ overnight and then centrifuged at $16900 \mathrm{~g}$ (Centrifuge $4795418 \mathrm{R}$, Eppendorf) for $30 \mathrm{~min}$ at $4{ }^{\circ} \mathrm{C}$. Supernatants were recovered and protein concentration of 480 LG lysates or collected tears were quantified using the BCA protein assay kit (Thermo Fisher 481 Scientific, reference 10678484, France). Fourty and $8 \mu \mathrm{g}$ of proteins from LG lysates and collected 482 tears respectively were loaded on any kD precast polyacrylamide gels (Mini-Protean® TGXTM gels, 483 Bio Rad, reference 4568124, France). Proteins were transferred to nitrocellulose membranes 484 (Trans-Blot Turbo Mini $0.2 \mu \mathrm{m}$ Nitrocellulose Transfer Pack, Bio Rad, reference 1704158, France) 
485 through semi-dry transfer process (Bio Rad Trans-Blot Turbo system). Membranes were incubated

486 with the REVERTTM total protein stain solution (LI-COR Biosciences, reference 926-11015,

487 France) to record the overall amount of protein per well. They were then blocked for $1 \mathrm{~h}$ at room 488 temperature using Intercept® blocking buffer (LI-COR Biosciences, reference 927-60001, France).

489 They were incubated with the following primary antibodies overnight at $4{ }^{\circ} \mathrm{C}$ in $\mathrm{LI}-\mathrm{COR}$ blocking 490 buffer: chicken anti-GFP (Aves Labs, reference GFP-1020, 1/2000) or rabbit mNGF (Abcam, 491 reference Ab52918, 1/500). Following three washes with TBS containing $0.1 \%$ Tween (TBST) for $49215 \mathrm{~min}$, secondary antibodies were incubated at a 1/15000 dilution in LI-COR blocking buffer: 493 donkey anti chicken IR Dye 800CW (LI-COR Biosciences, reference 926-32218) or donkey anti 494 rabbit IR Dye 800CW (LI-COR Biosciences, reference 926-32213). After three washes in TBST for $49515 \mathrm{~min}$, images were acquired with an Odyssey CLX LI-COR Imaging System (LI-COR 496 Biosciences, France) and the quantifications were performed with Image Studio lite software 497 (version 5.2). The GFP and the mNGF protein levels were both normalized to the total amount of 498 protein loaded per well. The results are expressed as the mean \pm SD.

\section{AAV2/9 biodistribution study}

501 LG, liver and heart from AAV2/9-CAG-mNGF-injected mice $\left(10^{11} \mathrm{vg} / \mathrm{LG}, n=7\right)$ were collected one 502 month post injection in DNA-free, RNAse/DNAse-free and PCR inhibitor-free certified microtubes. 503 Tissue samples were collected immediately after sacrifice, snap-frozen in liquid nitrogen and 504 stored at $-80{ }^{\circ} \mathrm{C}$ in conditions that minimize cross-contamination and avoid qPCR inhibition. 505 Extraction of genomic DNA (gDNA) from tissues using the Gentra Puregene kit (Qiagen, reference 506 158445, France) and Tissue Lyser II (Qiagen, reference 85300, France) was performed in 507 accordance with the manufacturer's recommendations. Vector genome copy number was 508 determined using a primer/FAM-TAMRA probe combination designed to amplify a specific region 509 of the $B G H$ transgene (BGH forward: TCTAGTTGCCAGCCATCTGTTGT, $B G H$ reverse: 510 TGGGAGTGGCACCTTCCA, BGH probe: FAM-TCCCCCGTGCCTTCCTTGACC-TAMRA). qPCR 511 analyses were conducted on a StepOne Plus apparatus (Applied Biosystems $\AA$, Thermo Fisher 512 Scientific, France) using 50 ng of gDNA in triplicates and the following cycling conditions: 
513 denaturation step $\left(20 \mathrm{sec}, 95^{\circ} \mathrm{C}\right)$ followed by a total of 45 cycles $\left(1 \mathrm{sec}, 95^{\circ} \mathrm{C} ; 20 \mathrm{sec}, 60^{\circ} \mathrm{C}\right)$. All

514 reactions were performed in a final volume of $20 \mu \mathrm{l}$ containing template DNA, Premix Ex Taq 515 (Takara/Ozyme, reference RR390L, France), 0.4 $\mu \mathrm{l}$ of ROX reference Dye (Takara/ozyme, 516 reference RR390L, France), $0.2 \mu \mathrm{mol} / / \mathrm{l}$ of each primer and $0.1 \mu \mathrm{mol} / \mathrm{l}$ of Taqman® probe (Dual517 Labeled Probes,Sigma-Aldrich, France). Endogenous gDNA copy numbers were determined using 518 the following primers/FAM-BHQ1 probe combination, designed to amplify a specific region of the 519 murine Albumin sequence (murine Albumin forward: AACTGAAACTTTGGGAGGT, murine 520 Albumin reverse: GGAGCACTTCATTCTCTGAC, murine Albumin probe: FAM521 AGCTTGATGGTGTGAAGGAGAAAG-BHQ1). qPCR analyses were conducted on a C1000 touch 522 thermal cycler (Bio-Rad, France) using $50 \mathrm{ng}$ of gDNA in triplicates and the following cycling 523 conditions: denaturation step $\left(20 \mathrm{sec}, 95^{\circ} \mathrm{C}\right)$ followed by a total of 45 cycles $\left(3 \mathrm{sec}, 95^{\circ} \mathrm{C} ; 30 \mathrm{sec}\right.$, $52460^{\circ} \mathrm{C}$ ). All reactions were performed in a final volume of $20 \mu$ l containing template DNA, Premix Ex 525 Taq (Takara/Ozyme, reference RR390L,France), $0.25 \mu \mathrm{mol} / \mathrm{l}$ of each primer and $0.2 \mu \mathrm{mol} / \mathrm{l}$ of 526 Taqman® probe (Dual-Labeled Probes,Sigma-Aldrich, France). For each sample, threshold cycle 527 (Ct) values were compared with those obtained with different dilutions of linearized standard 528 plasmids (containing either the BGH expression cassette or the murine Albumin gene) using Bio529 Rad CFX Maestro 2.2 software (version version 5.2.008.0222) or StepOne software (version 2.3) 530 for the $\mathrm{C} 1000$ touch thermal cycler and the StepOne Plus apparatus respectively. The absence of 531 qPCR inhibition in the presence of gDNA was checked by analyzing $50 \mathrm{ng}$ of gDNA extracted from 532 tissue samples from two AAV2/9 empty capsid-injected control mice. Results were expressed in 533 vector genome copies per diploid genome $(\mathrm{vg} / \mathrm{dg})$ as the mean \pm SD. The lowest limit of 534 quantification (LLOQ) was determined as $0.001 \mathrm{vg} / \mathrm{dg}$. Only values of $\mathrm{vg} / \mathrm{dg}$ above the LLOQ 535 were presented.

536

\section{Von Frey test}

538 Corneal sensitivity was evaluated using von Frey filaments (Bioseb, reference bio-VF-M) as 539 previously described ${ }^{51}$. Filaments with define forces from 0.008 to $0.6 \mathrm{~g}$ were applied on the cornea 540 of an immobilized mouse until an eye-blink reflex was observed. Mice were habituated every day 
541 for five days. Von Frey test was performed on each cornea for two consecutive days (contralateral

542 side and injected side). The values obtained from these two days were averaged for each cornea.

543 As the values were represented in $\mathrm{g}$, we displayed them as $1 / \mathrm{g}$ to reflect the sensitivity. Results are

544 expressed as the mean \pm SD. Behavioural experiments and analysis were performed by the same

545 experimenter in single-blinded conditions throughout the study.

546

547 Statistical analysis

548 Data were analyzed with GraphPad Prism software (version 9.1.2, Prism, CA, USA) and expressed

549 as the mean \pm SD as indicated in the Figure legends. Statistical differences between mean values

550 were tested using Brown-Forsythe and Welch ANOVA tests followed by Dunnett's T3 multiple

551 comparisons test, repeated measures two-way ANOVA test followed by Sidak's or Tukey's multiple

552 comparisons test, repeated measure one-way ANOVA test followed by Dunnett's comparisons

553 test, Friedman's one-way ANOVA test followed by Dunn's multiple comparisons test or simple

554 linear regression as indicated in the Figure legends. Differences between values were considered

555 significant with: ${ }^{*} p<0.05$, ${ }^{* *} p<0.01,{ }^{* *} p<0.001,{ }^{* * * *} p<0.0001$. All exact $p$-values are

556 available in the Source Data File.

557

558 Data and materials availability

559 All data and materials are available upon request. The source data underlying Figures 3-7, 8A, 8B, 560 9B and Supplementary Figures S3-S5 are provided as a Source Data File. 


\section{Ethics declarations}

563 The authors declare no competing interests.

564

\section{Author contributions}

566 Conceptualization: B.G., F.M.; Methodology: B.G., L.M., L.H.; Validation: B.G., F.M.; Formal 567 analysis: B.G., L.H., F.M.; Investigation: B.G., L.M., C.A., L.H., N.F., A.K., A.P., C.L.G., V.B., F.M. 568 Data analysis: B.G., F.M.; Writing-Original Draft: B.G., F.M.; Writing-Review and Editing: all 569 authors; Supervision: F.M.; Project administration: F.M.; Funding acquisition: C.D., F.M.

570

\section{Acknowledgments}

572 The authors thank the different technical platforms of the Institute for Neurosciences of Montpellier, 573 especially the RAM-Neuro, animal core facility supervised by Denis Greuet, the imaging facility $574 \mathrm{MRI}$, member of the national infrastructure France-Biolmaging infrastructure supported by the 575 French National Research Agency (ANR-10-INBS-04, «Investments for the future»), and the CPV 576 vector core and Preclinical Analytics Core from the TarGeT lab, INSERM UMR 1089, Nantes 577 University (https://umr1089.univ-nantes.fr/facilities-cores/). This research was supported by the 578 ATIP-Avenir program, Inserm-Transfert, Inserm, Région Occitanie, University of Montpellier, and 579 Retina France. 


\section{References}

582 1. Kalha, S., Shrestha, B., Sanz Navarro, M., Jones, K.B., Klein, O.D., and Michon, F. (2018).

583 Bmi1+ Progenitor Cell Dynamics in Murine Cornea During Homeostasis and Wound Healing.

$584 \quad$ Stem Cells 36, 562-573.

2. Zieske, J.D. (2004). Corneal development associated with eyelid opening. Int. J. Dev. Biol. 48, 903-911.

3. Kuony, A., Ikkala, K., Kalha, S., Magalhães, A.C., Pirttiniemi, A., and Michon, F. (2019). Ectodysplasin-A signaling is a key integrator in the lacrimal gland-cornea feedback loop. Development 146.

4. Müller, L.J., Marfurt, C.F., Kruse, F., and Tervo, T.M.T. (2003). Corneal nerves: structure, contents and function. Experimental Eye Research 76, 521-542. (StatPearls Publishing). Corneal injury: Clinical and molecular aspects. Experimental Eye Research 186, 107709. Inflammation in Sjögren’s Syndrome-Related Dry Eye Disease. IJMS 22, 11820. Lacrimal Glands. Immune Netw 19, e3. 
603 10. Lambiase, A., and Sacchetti, M. (2014). Diagnosis and management of neurotrophic keratitis. 604 OPTH, 571.

605 11. Mastropasqua, L., Massaro-Giordano, G., Nubile, M., and Sacchetti, M. (2017). 606 Understanding the Pathogenesis of Neurotrophic Keratitis: The Role of Corneal Nerves: THE 607 ROLE OF CORNEAL NERVES IN NEUROTROPHIC KERATITIS. J. Cell. Physiol. 232, 717$608 \quad 724$.

610 of diabetes on corneal nerve morphology and ocular surface integrity. The Ocular Surface 16, $611 \quad 45-57$.

612 13. Han, S.B., Yang, H.K., and Hyon, J.Y. (2018). Influence of diabetes mellitus on anterior 613 segment of the eye. CIA Volume 14, 53-63.

614 14. Yeh, S.-I., Chu, T.-W., Cheng, H.-C., Wu, C.-H., and Tsao, Y.-P. (2020). The Use of 615 Autologous Serum to Reverse Severe Contact Lens-induced Limbal Stem Cell Deficiency. 616 Cornea 39, 736-741.

617 15. Kanu, L.N., and Ciolino, J.B. (2021). Nerve Growth Factor as an Ocular Therapy: 618 Applications, Challenges, and Future Directions. Seminars in Ophthalmology 36, 224-231.

619 16. Eaton, A.M., Gordon, G.M., Konowal, A., Allen, A., Allen, M., Sgarlata, A., Gao, G., 620 Wafapoor, H., and Avery, R.L. (2015). A novel eye drop application monitor to assess patient 621 compliance with a prescribed regimen: a pilot study. Eye 29, 1383-1391.

622 17. Wang, D., Tai, P.W.L., and Gao, G. (2019). Adeno-associated virus vector as a platform for 623 gene therapy delivery. Nat Rev Drug Discov 18, 358-378. 
624 18. Mendell, J.R., Al-Zaidy, S.A., Rodino-Klapac, L.R., Goodspeed, K., Gray, S.J., Kay, C.N., 625 Boye, S.L., Boye, S.E., George, L.A., Salabarria, S., et al. (2021). Current Clinical Applications 626 of In Vivo Gene Therapy with AAVs. Molecular Therapy 29, 464-488.

627

628

629

630

631

19. Kuzmin, D.A., Shutova, M.V., Johnston, N.R., Smith, O.P., Fedorin, V.V., Kukushkin, Y.S., van der Loo, J.C.M., and Johnstone, E.C. (2021). The clinical landscape for AAV gene therapies. Nat Rev Drug Discov 20, 173-174.

20. Maguire, A.M., Russell, S., Chung, D.C., Yu, Z.-F., Tillman, A., Drack, A.V., Simonelli, F., Leroy, B.P., Reape, K.Z., High, K.A., et al. (2021). Durability of Voretigene Neparvovec for Biallelic RPE65-Mediated Inherited Retinal Disease. Ophthalmology 128, 1460-1468.

21. Day, J.W., Finkel, R.S., Chiriboga, C.A., Connolly, A.M., Crawford, T.O., Darras, B.T., Iannaccone, S.T., Kuntz, N.L., Peña, L.D.M., Shieh, P.B., et al. (2021). Onasemnogene abeparvovec gene therapy for symptomatic infantile-onset spinal muscular atrophy in patients with two copies of SMN2 (STR1VE): an open-label, single-arm, multicentre, phase 3 trial. The Lancet Neurology 20, 284-293.

22. Auricchio, A., Smith, A.J., and Ali, R.R. (2017). The Future Looks Brighter After 25 Years of Retinal Gene Therapy. Human Gene Therapy 28, 982-987.

23. Mohan, R.R., Martin, L.M., and Sinha, N.R. (2021). Novel insights into gene therapy in the cornea. Experimental Eye Research 202, 108361.

24. Rocha, E.M., Di Pasquale, G., Riveros, P.P., Quinn, K., Handelman, B., and Chiorini, J.A. (2011). Transduction, tropism, and biodistribution of AAV vectors in the lacrimal gland. Invest Ophthalmol Vis Sci 52, 9567-9572. 
645 25. Kuony, A., and Michon, F. (2017). Epithelial Markers aSMA, Krt14, and Krt19 Unveil

646 Elements of Murine Lacrimal Gland Morphogenesis and Maturation. Front Physiol 8, 739.

647 26. Klenkler, B., Sheardown, H., and Jones, L. (2007). Growth Factors in the Tear Film: Role in 648 Tissue Maintenance, Wound Healing, and Ocular Pathology. The Ocular Surface 5, 228-239.

649 27. Bax, B., Blundell, T.L., Murray-Rust, J., and McDonald, N.Q. (1997). Structure of mouse 7S

650 NGF: a complex of nerve growth factor with four binding proteins. Structure 5, 1275-1285.

651 28. Yan, R., Yalinca, H., Paoletti, F., Gobbo, F., Marchetti, L., Kuzmanic, A., Lamba, D., 652 Gervasio, F.L., Konarev, P.V., Cattaneo, A., et al. (2019). The Structure of the Pro-domain of 653 Mouse proNGF in Contact with the NGF Domain. Structure 27, 78-89.e3.

654 29. Young, T.L. (2003). Ophthalmic genetics/inherited eye disease: Current Opinion in 655 Ophthalmology 14, 296-303.

656 30. Latta, L., Figueiredo, F.C., Ashery-Padan, R., Collinson, J.M., Daniels, J., Ferrari, S., 657 Szentmáry, N., Solá, S., Shalom-Feuerstein, R., Lako, M., et al. (2021). Pathophysiology of 658 aniridia-associated keratopathy: Developmental aspects and unanswered questions. The Ocular 659 Surface 22, 245-266.

660 31. Abdul-Al, M., Kyeremeh, G.K., Saeinasab, M., Heidari Keshel, S., and Sefat, F. (2021). Stem 661 Cell Niche Microenvironment: Review. Bioengineering 8, 108.

662 32. Hudry, E., and Vandenberghe, L.H. (2019). Therapeutic AAV Gene Transfer to the Nervous 663 System: A Clinical Reality. Neuron 101, 839-862.

664 33. Bordet, T., and Behar-Cohen, F. (2019). Ocular gene therapies in clinical practice: viral 665 vectors and nonviral alternatives. Drug Discovery Today 24, 1685-1693. 
666 34. Ochakovski, G.A., Bartz-Schmidt, K.U., and Fischer, M.D. (2017). Retinal Gene Therapy: 667 Surgical Vector Delivery in the Translation to Clinical Trials. Front. Neurosci. 11.

668 35. Schuster, D.J., Dykstra, J.A., Riedl, M.S., Kitto, K.F., Belur, L.R., McIvor, R.S., Elde, R.P., 669 Fairbanks, C.A., and Vulchanova, L. (2014). Biodistribution of adeno-associated virus serotype 9 670 (AAV9) vector after intrathecal and intravenous delivery in mouse. Front. Neuroanat. 8.

671 36. Colle, M.-A., Piguet, F., Bertrand, L., Raoul, S., Bieche, I., Dubreil, L., Sloothaak, D., 672 Bouquet, C., Moullier, P., Aubourg, P., et al. (2010). Efficient intracerebral delivery of AAV5 673 vector encoding human ARSA in non-human primate. Human Molecular Genetics 19, 147-158.

674 37. Gautier, B., Hajjar, H., Soares, S., Berthelot, J., Deck, M., Abbou, S., Campbell, G., Ceprian, 675 M., Gonzalez, S., Fovet, C.-M., et al. (2021). AAV2/9-mediated silencing of PMP22 prevents the 676 development of pathological features in a rat model of Charcot-Marie-Tooth disease 1 A. Nat 677 Commun 12, 2356.

678 38. Powell, S.K., and Gray, S.J. (2015). Viral Expression Cassette Elements to Enhance 679 Transgene Target Specificity and Expression in Gene Therapy. 15.

680 39. Gray, S.J., Foti, S.B., Schwartz, J.W., Bachaboina, L., Taylor-Blake, B., Coleman, J., Ehlers, 681 M.D., Zylka, M.J., McCown, T.J., and Samulski, R.J. (2011). Optimizing Promoters for 682 Recombinant Adeno-Associated Virus-Mediated Gene Expression in the Peripheral and Central 683 Nervous System Using Self-Complementary Vectors. Human Gene Therapy 22, 1143-1153.

684 40. Grimm, D., Streetz, K.L., Jopling, C.L., Storm, T.A., Pandey, K., Davis, C.R., Marion, P., 685 Salazar, F., and Kay, M.A. (2006). Fatality in mice due to oversaturation of cellular 686 microRNA/short hairpin RNA pathways. Nature 441, 537-541. 
687 41. Auricchio, A. (2001). Exchange of surface proteins impacts on viral vector cellular specificity 688 and transduction characteristics: the retina as a model. Human Molecular Genetics 10, 30756893081.

690 42. Hinderer, C., Katz, N., Buza, E.L., Dyer, C., Goode, T., Bell, P., Richman, L.K., and Wilson, 691 J.M. (2018). Severe Toxicity in Nonhuman Primates and Piglets Following High-Dose 692 Intravenous Administration of an Adeno-Associated Virus Vector Expressing Human SMN. 693 Human Gene Therapy 29, 285-298.

694 43. Zincarelli, C., Soltys, S., Rengo, G., and Rabinowitz, J.E. (2008). Analysis of AAV Serotypes 695 1-9 Mediated Gene Expression and Tropism in Mice After Systemic Injection. Molecular 696 Therapy $16,1073-1080$.

697 44. Bailey, R.M., Armao, D., Nagabhushan Kalburgi, S., and Gray, S.J. (2018). Development of 698 Intrathecal AAV9 Gene Therapy for Giant Axonal Neuropathy. Molecular Therapy - Methods \& 699 Clinical Development 9, 160-171.

700 45. Brown, B.D., Venneri, M.A., Zingale, A., Sergi, L.S., and Naldini, L. (2006). Endogenous 701 microRNA regulation suppresses transgene expression in hematopoietic lineages and enables 702 stable gene transfer. Nat Med 12, 585-591.

703 46. Dhouailly, D. (2009). A new scenario for the evolutionary origin of hair, feather, and avian 704 scales. Journal of Anatomy 214, 587-606.

705 47. Colella, P., Ronzitti, G., and Mingozzi, F. (2018). Emerging Issues in AAV-Mediated In Vivo 706 Gene Therapy. Molecular Therapy - Methods \& Clinical Development 8, 87-104. 
707 48. Manni, L., Rocco, M.L., Bianchi, P., Soligo, M., Guaragna, M., Barbaro, S.P., and Aloe, L.

708 (2013). Nerve growth factor: basic studies and possible therapeutic applications. Growth Factors $709 \quad 31,115-122$.

710 49. Ayuso, E., Mingozzi, F., and Bosch, F. (2010). Production, Purification and Characterization 711 of Adeno-Associated Vectors. CGT 10, 423-436.

712 50. Kalha, S., Kuony, A., and Michon, F. (2018). Corneal Epithelial Abrasion with Ocular Burr 713 As a Model for Cornea Wound Healing. J Vis Exp.

714 51. Fakih, D., Zhao, Z., Nicolle, P., Reboussin, E., Joubert, F., Luzu, J., Labbé, A., Rostène, W., 715 Baudouin, C., Mélik Parsadaniantz, S., et al. (2019). Chronic dry eye induced corneal 716 hypersensitivity, neuroinflammatory responses, and synaptic plasticity in the mouse trigeminal 717 brainstem. J Neuroinflammation 16, 268.

718

719

720 


\section{Figures legends}

722 Figure 1. AAV2/9 and AAV2/5-CAG-GFP transduce widely the murine LG. Representative 723 images of LG longitudinal sections show GFP protein (green) following a single injection of AAV2/9 724 or AAV2/5-CAG-GFP into murine LG, when compared to AAV2/9 or AAV2/5 empty capsid-injected 725 mice respectively ( $10^{10} \mathrm{vg} / \mathrm{LG}$ in $3 \mu \mathrm{l}, n=3$ mice per group). Mice were sacrificed one month post 726 injection. Nuclei are counterstained with Hoechst 33342 (white). Scale bar: $500 \mu \mathrm{m}$.

Figure 2. AAV2/9 and AAV2/5-CAG-GFP transduce LG acinar and ductal cells. Representative images of LG longitudinal sections show GFP protein (green) in acinar cells immunostained for E-

730 Cadh (red, A) and in ductal cells immunostained for Krt19 (red, B) after a single injection of 731 AAV2/9 or AAV2/5-CAG-GFP into murine LG, when compared to AAV2/9 or AAV2/5 empty capsid732 injected mice respectively $\left(10^{10} \mathrm{vg} / \mathrm{LG}\right.$ in $3 \mu \mathrm{l}, n=3$ mice per group). Mice were sacrificed one 733 month post injection. Nuclei are counterstained with Hoechst 33342 (white). Scale bar: $10 \mu \mathrm{m}$ (A) 734 and $20 \mu \mathrm{m}(\mathbf{B})$.

735

Figure 3. GFP is detected in AAV2/9 and AAV2/5-CAG-GFP-injected LG and dosed by

Western blot. A. Representative Western blot image showing GFP level (green) and total protein as loading control (white) in murine LG lysates from AAV2/9 or AAV2/5-CAG-GFP-injected mice, when compared to AAV2/9 or AAV2/5 empty capsid-injected mice respectively $\left(10^{10} \mathrm{vg} / \mathrm{LG}\right.$ in 3 $\mu l)$. Mice were sacrificed one month post injection. B. Quantification of GFP / total protein ratio in

741 LG lysates ( $n=3$ mice per group). Results are expressed as the mean \pm SD. Statistical analysis 742 using Brown-Forsythe and Welch ANOVA tests followed by Dunnett's T3 multiple comparisons 743 test. ${ }^{* *} p=0.0026$ between AAV2/9 empty capsid and AAV2/9 CAG-GFP groups, ${ }^{* *} p=0.0077$ 744 between AAV2/5 empty capsid and AAV2/5-CAG-GFP groups, ${ }^{* *} p=0.0018$ between AAV2/9745 CAG-GFP and AAV2/5-CAG-GFP groups; ns, not significant; A. U., arbitrary units. Source data are 746 provided as a Source Data file. 
Figure 4. Injections of AAV2/9 and AAV2/5-CAG-mNGF into murine LG significantly increase

750 the level of $\mathrm{mNGF}$ in tears. mNGF protein levels $(\mathrm{ng} / \mathrm{ml}$ ) were measured by ELISA in the tears 751 of AAV2/9 or AAV2/5-CAG-mNGF-injected mice, when compared to AAV2/9 or AAV2/5 empty 752 capsid-injected mice, one month post injection respectively $\left(10^{10} \mathrm{vg} / \mathrm{LG}\right.$ in $3 \mu \mathrm{l}, n=5$ mice per 753 group). Results are expressed as the mean \pm SD. Statistical analysis using Brown-Forsythe and 754 Welch ANOVA tests followed by Dunnett's T3 multiple comparisons test. ${ }^{* *} p=0.0065$ between 755 AAV2/9 empty capsid and AAV2/9-CAG-mNGF groups, ${ }^{*} p=0.0464$ between AAV2/5 empty 756 capsid and AAV2/5-CAG-mNGF groups, ${ }^{* *} p=0.0085$ between AAV2/9-CAG-mNGF and AAV2/5757 CAG-mNGF groups. Source data are provided as a Source Data file.

758

Figure 5. Injections of AAV2/9 and AAV2/5-CAG-mNGF into murine LG significantly increase

760 the level of total, pro and mature mNGF in tears. mNGF protein levels were analyzed by 761 Western blot experiments in the tears of AAV2/9 or AAV2/5-CAG-mNGF-injected mice, when 762 compared to AAV2/9 or AAV2/5 empty capsid-injected mice, one month post injection respectively $763\left(10^{10} \mathrm{vg} / \mathrm{LG}\right.$ in $3 \mu \mathrm{l}, n=5$ mice per group). A. Representative Western blot image showing mNGF 764 level (green) and total protein as loading control (white) in the tears of injected mice. Quantification 765 of total mNGF (B), pro mNGF (C) and mature mNGF (D) protein levels in the tears of injected mice 766 Results are expressed as the mean \pm SD. Statistical analysis using Brown-Forsythe and Welch 767 ANOVA tests followed by Dunnett's T3 multiple comparisons test. B. ${ }^{* \star} p=0.0091$ between AAV2/9 768 empty capsid and AAV2/9-CAG-mNGF groups, * $p=0.0397$ between AAV2/5 empty capsid and 769 AAV2/5-CAG-mNGF groups, * $p=0.0235$ between AAV2/9-CAG-mNGF and AAV2/5-CAG-mNGF 770 groups. C. ${ }^{* *} p=0.0027$ between AAV2/9 empty capsid and AAV2/9-CAG-mNGF groups, $771{ }^{*} p=0.0363$ between AAV2/5 empty capsid and AAV2/5-CAG-mNGF groups, * $p=0.0127$ 772 between AAV2/9-CAG-mNGF and AAV2/5-CAG-mNGF groups. D. * $p=0.0162$ between AAV2/9 773 empty capsid and AAV2/9-CAG-mNGF groups * $p=0.0388$ between AAV2/5 empty capsid and 774 AAV2/5-CAG-mNGF groups, * $p=0.0368$ between AAV2/9-CAG-mNGF and AAV2/5-CAG-mNGF 775 groups. ns, not significant; A. U., arbitrary units. Source data are provided as a Source Data file. 
Figure 6. Dose response and kinetic studies after injection of AAV2/9-CAG-mNGF into murine LG reveal a high and long-lasting secretion of $\mathrm{mNGF}$ in tears. For the dose response study, mNGF levels were analyzed by ELISA in the tears of AAV2/9-CAG-mNGF-injected mice by 780 increasing dose of vectors $\left(10^{9}, 10^{10}\right.$, and $10^{11} \mathrm{vg} / \mathrm{LG}$ in $3 \mu \mathrm{l}, \mathrm{n}=5$ mice per dose), when 781 compared to the tears of AAV2/9 empty capsid-injected mice one month post injection. For the 782 kinetic study, mNGF levels were analyzed by ELISA in the tears of AAV2/9-CAG-mNGF-injected 783 mice $\left(10^{11} \mathrm{vg} / \mathrm{LG}\right.$ in $\left.3 \mu \mathrm{l}\right)$, when compared to the tears of AAV2/9 empty capsid-injected mice, 1 day before injection and at 7, 30,60, 120 and 180 days post injection ( $n=3$ and 5 for AAV2/9 empty capsid-injected mice and AAV2/9-CAG-mNGF-injected mice respectively). A. Quantification by ELISA of mNGF level (ng / $\mathrm{ml})$ in the tears of injected mice at the indicated doses. Results are expressed as the mean \pm SD. Statistical analysis using Brown-Forsythe and Welch ANOVA tests followed by Dunnett's T3 multiple comparisons test. ${ }^{*} p=0.0107$ and ${ }^{*} p=0.0341$ between the doses $10^{9}$ and $10^{10} \mathrm{vg} / \mathrm{LG}$ and between the doses $10^{10}$ and $10^{11} \mathrm{vg} / \mathrm{LG}$ respectively. ns, not significant. B. Quantification by ELISA of $\mathrm{mNGF}$ level in tears $(\mathrm{ng} / \mathrm{ml})$ at the indicated days post

791 injection. Statistical analysis using repeated measures two-way ANOVA test followed by Sidak's 792 multiple comparisons test. ${ }^{* * *} p=0.0009$, * $p=0.0225,{ }^{*} p=0.0268,{ }^{*} p=0.0260$, between 793 AAV2/9 empty capsid and AAV2/9-CAG-mNGF groups at 7, 30, 60, 120 and 180 days post injection respectively. ns, not significant. Source data are provided as a Source Data file.

Figure 7. Biodistribution analysis after injection of AAV2/9-CAG-mNGF into murine LG reveals a limited diffusion to peripheral organs. AAV2/9-CAG-mNGF was injected into the murine LG $\left(10^{11} \mathrm{vg} / \mathrm{LG}\right.$ in $3 \mu \mathrm{l}, n=7$ mice $)$. One month post injection tears were collected and mNGF level was quantified by ELISA. At the same time, mice were sacrificed, LG, liver and heart were collected and analyzed by qPCR. A. Quantification of the transduction rate expressed in 801 vector genome / diploid genome ( $\mathrm{vg} / \mathrm{dg}$ ) above the lowest limit of quantification (LLOQ) ( $n=7$ for 802 LG and liver samples, $n=6$ for heart samples). Results are expressed as the mean \pm SD. 803 Statistical analysis using Brown-Forsythe and Welch ANOVA tests followed by Dunnett's T3 
804 multiple comparisons test. ${ }^{* \star * *} p<0.0001$ between LG and liver, ${ }^{* * * *} p<0.0001$ between LG and

805 heart, ${ }^{*} p=0.0226$ between liver and heart. B. Graph showing the statistical linear regression (red 806 line) for each mouse between mNGF level ( $\mathrm{ng} / \mathrm{ml}$ ) in tears and $\mathrm{vg} / \mathrm{dg}$ in $\mathrm{LG}$. $\mathrm{R}^{2}, \mathrm{R}$ squared. 807 Source data are provided as a Source Data file.

808

809 Figure 8. Injection of AAV2/9-CAG-mNGF into murine LG does not alter tear protein 810 concentration nor corneal integrity. AAV2/9 empty capsid or AAV2/9-CAG-mNGF were injected 811 into murine $\mathrm{LG}\left(10^{11} \mathrm{vg} / \mathrm{LG}\right.$ in $3 \mu \mathrm{l}, n=3$ and 5 mice respectively). The volume of tears $(\mathbf{A})$, the 812 protein concentration in tears $(\mathbf{B})$ and the corneal integrity $(\mathbf{C})$ were assessed 1 day before 813 injection and 7, 30, 60, 120 days and 180 post injection. A. Graph showing the volume of tears $(\mu \mathrm{l} /$ $814 \mathrm{~min}$ ) collected before injection and at the indicated days post injection. Results are expressed as 815 the mean \pm SD. Statistical analysis using repeated measures two-way ANOVA test followed by 816 Sidak's multiple comparisons test. ${ }^{* *} p=0.0036,{ }^{* * *} p<0.0001,{ }^{* *} p=0.0057,{ }^{*} p=0.0246$ 817 between AAV2/9 empty capsid and AAV2/9 CAG-mNGF groups at 7, 30, 60 and 120 days post 818 injection respectively. ns, not significant. B. Graph showing the protein concentration in tears 819 (mg/ml) collected before injection and at the indicated days post injection. Results are expressed 820 as the mean \pm SD. Statistical analysis using repeated measures two-way ANOVA test followed by

821 Sidak's multiple comparisons test. ns, not significant. C. Eye images obtained from the fluorescein 822 stain test. Fluorescein signal highlights an abraded cornea in bright green, while an intact cornea 823 remains dark. Source data are provided as a Source Data file.

824

Figure 9. Injection of AAV2/9-CAG-mNGF into murine LG does not affect corneal innervation

826 nor sensitivity. A. Representative images showing corneal innervation. Whole corneas were 827 immunostained for $\beta$ III-tubulin (green) following a single injection of AAV2/9-CAG-mNGF into 828 murine LG, when compared to AAV2/9 empty capsid-injected mice $\left(10^{11} \mathrm{vg} / \mathrm{LG}\right.$ in $3 \mu \mathrm{l}, n=3$ mice 829 per group). Mice were sacrificed two weeks post injection. Nuclei are counterstained with 830 BioTracker NIR694 (magenta). Scale bar: $500 \mu \mathrm{m}$. B. Quantification of the corneal sensitivity 
$831(1 / \mathrm{g})$ measured by von Frey test performed both on the contralateral side and the injected side of 832 AAV2/9 empty capsid (white plots, $n=7$ ) or AAV2/9-CAG-mNGF (red plots, $n=5$ )-injected mice. 833 Von Frey analysis was performed two weeks post injection. Results are expressed as the mean \pm 834 SD. Statistical analysis using two-way ANOVA test followed by Sidak's multiple comparisons test. 835 ns, not significant. Source data are provided as a Source Data file.

836 

A
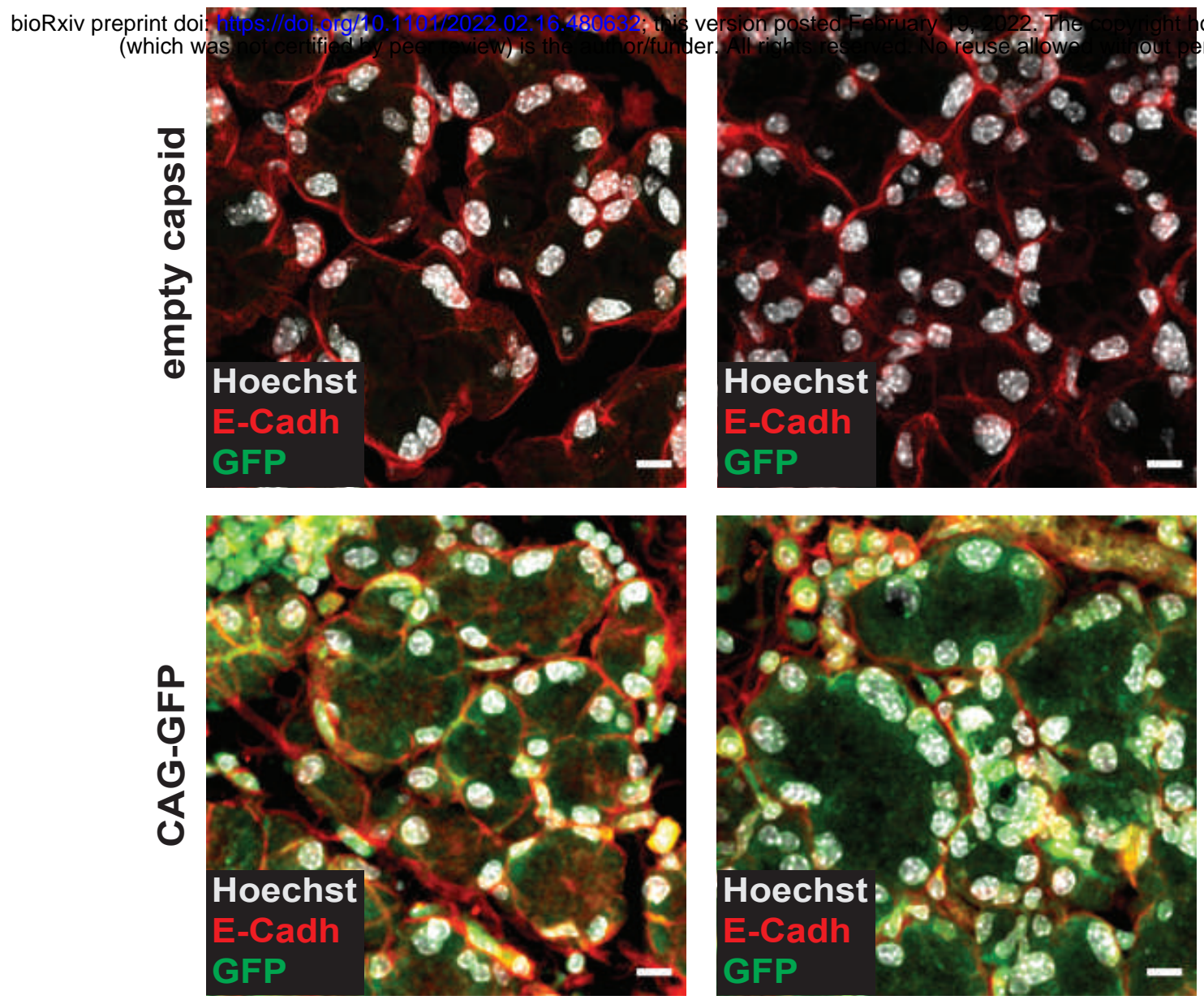

B

AAV2/9
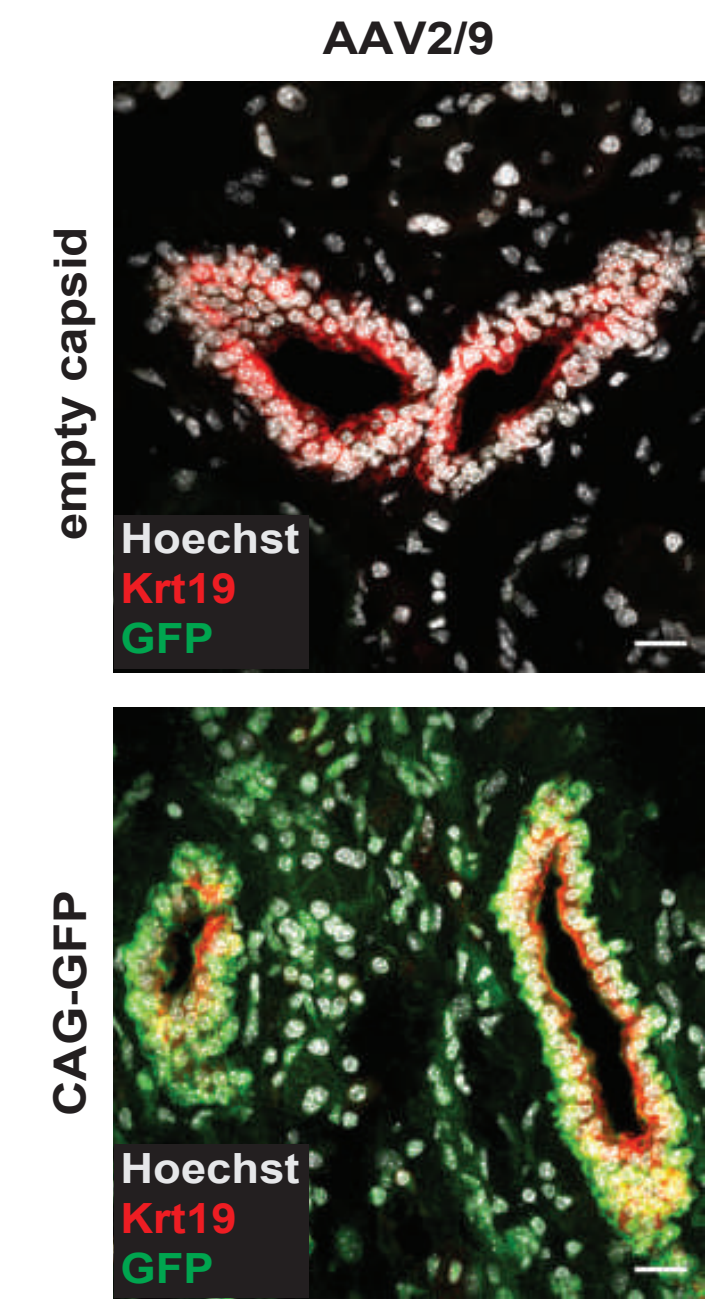

AAV2/5
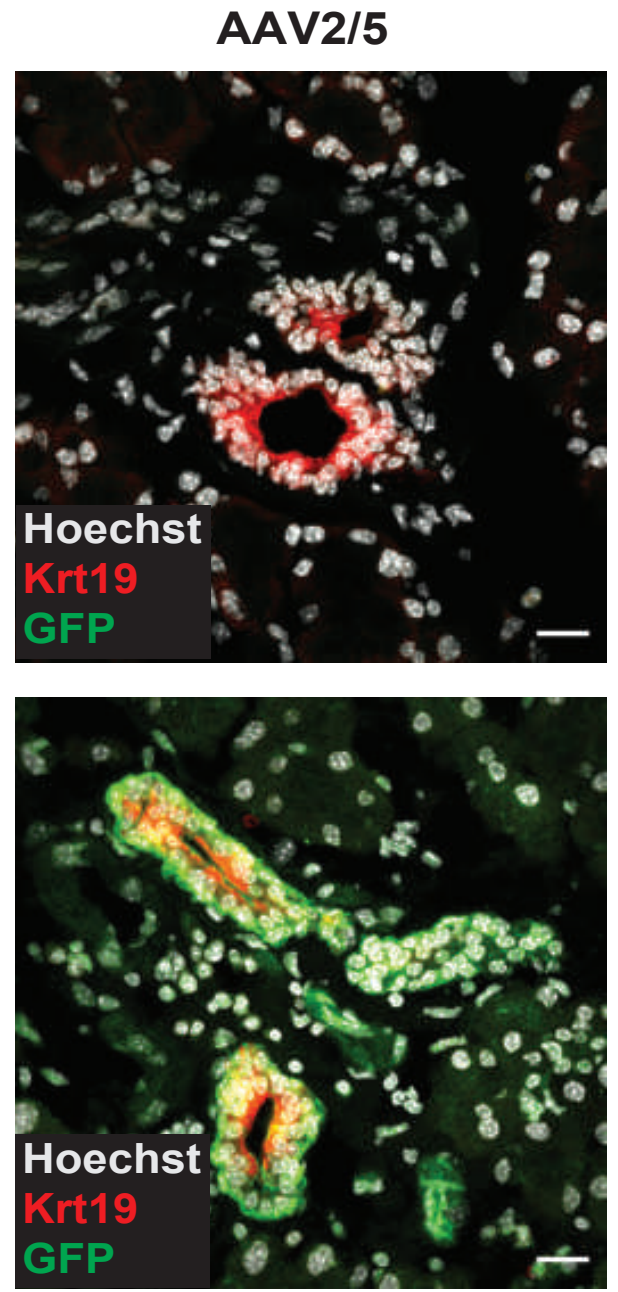

older for this preprint ermission. 


\section{Figure 3}

A bioRxiv preprint doi: https://doi.org/10.1101/2022.02.16.480632; this version posted February 19, 2022. The copyright holder for this preprint
(which was not certified by peer review) is the author/funder. All rights reserved. No reuse allowed without permission.

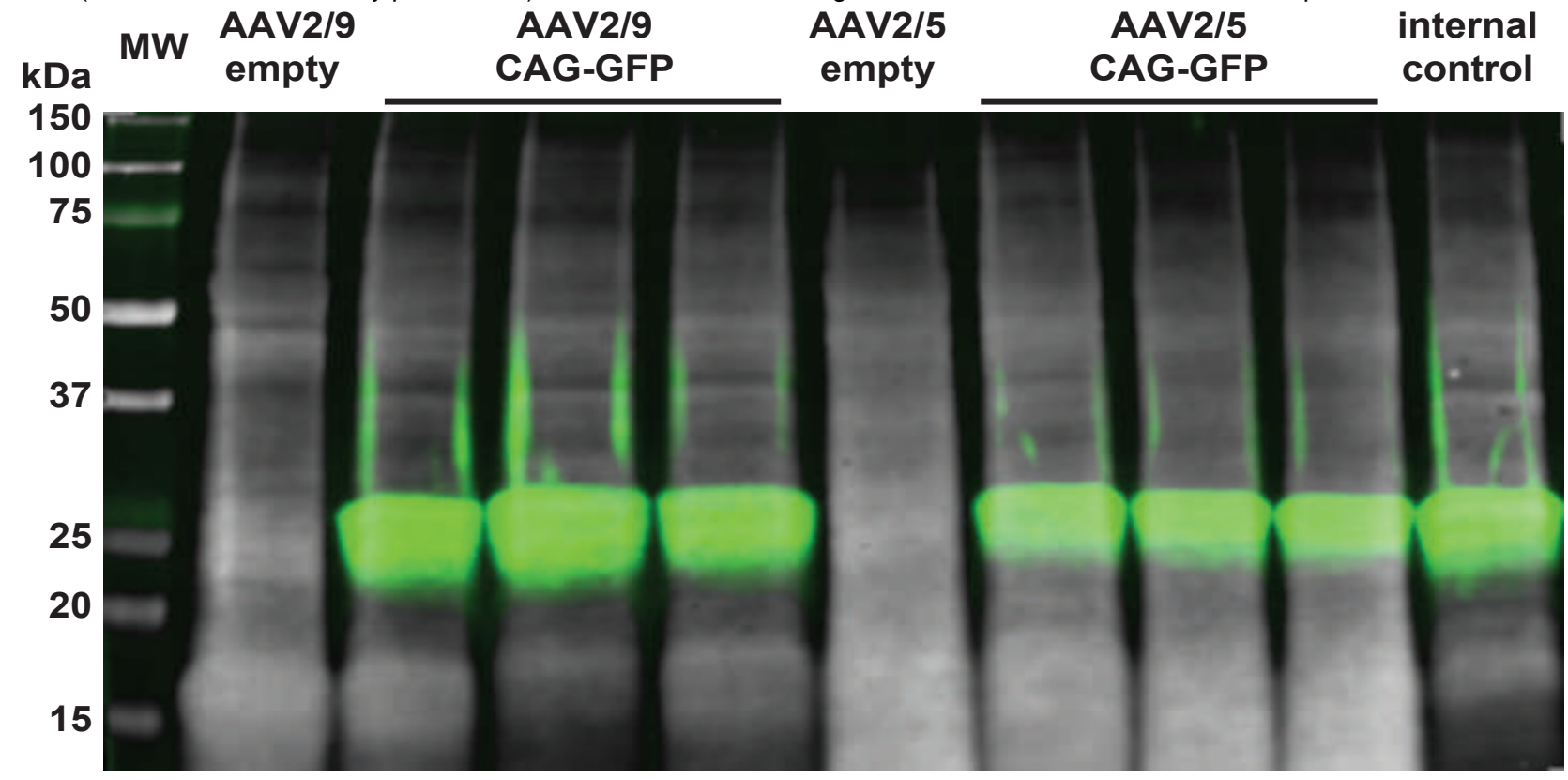

B

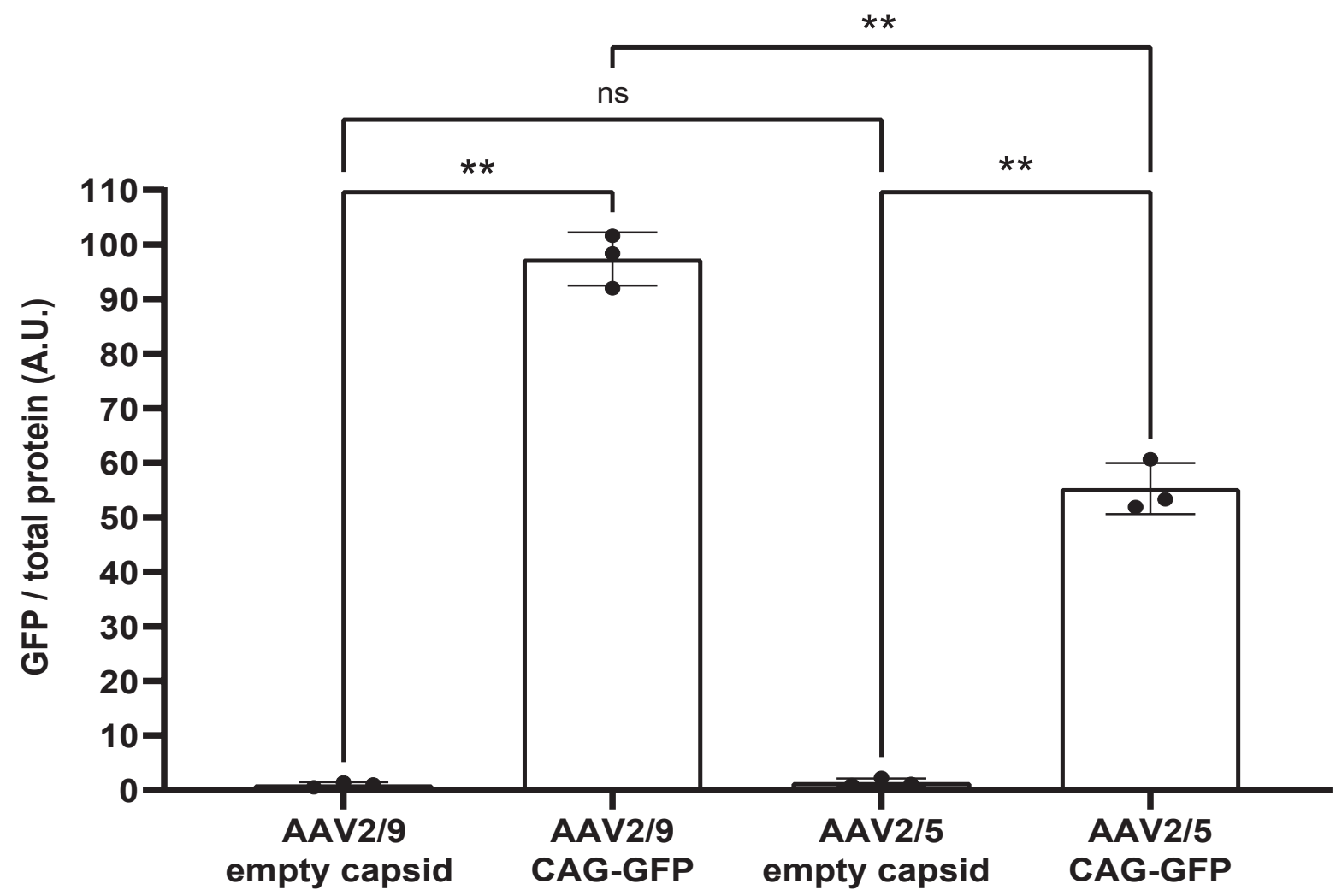




\section{Figure 4}

**

bioRxiv preprint doi: https://doi.org/10.1101/2022.02.16 400032, this versiompostedFetruary 19, 2022. The copyright holder for this preprint (which was not certified by peer review) is the author/funder. All rights reserved. No reuse allowed without permission.

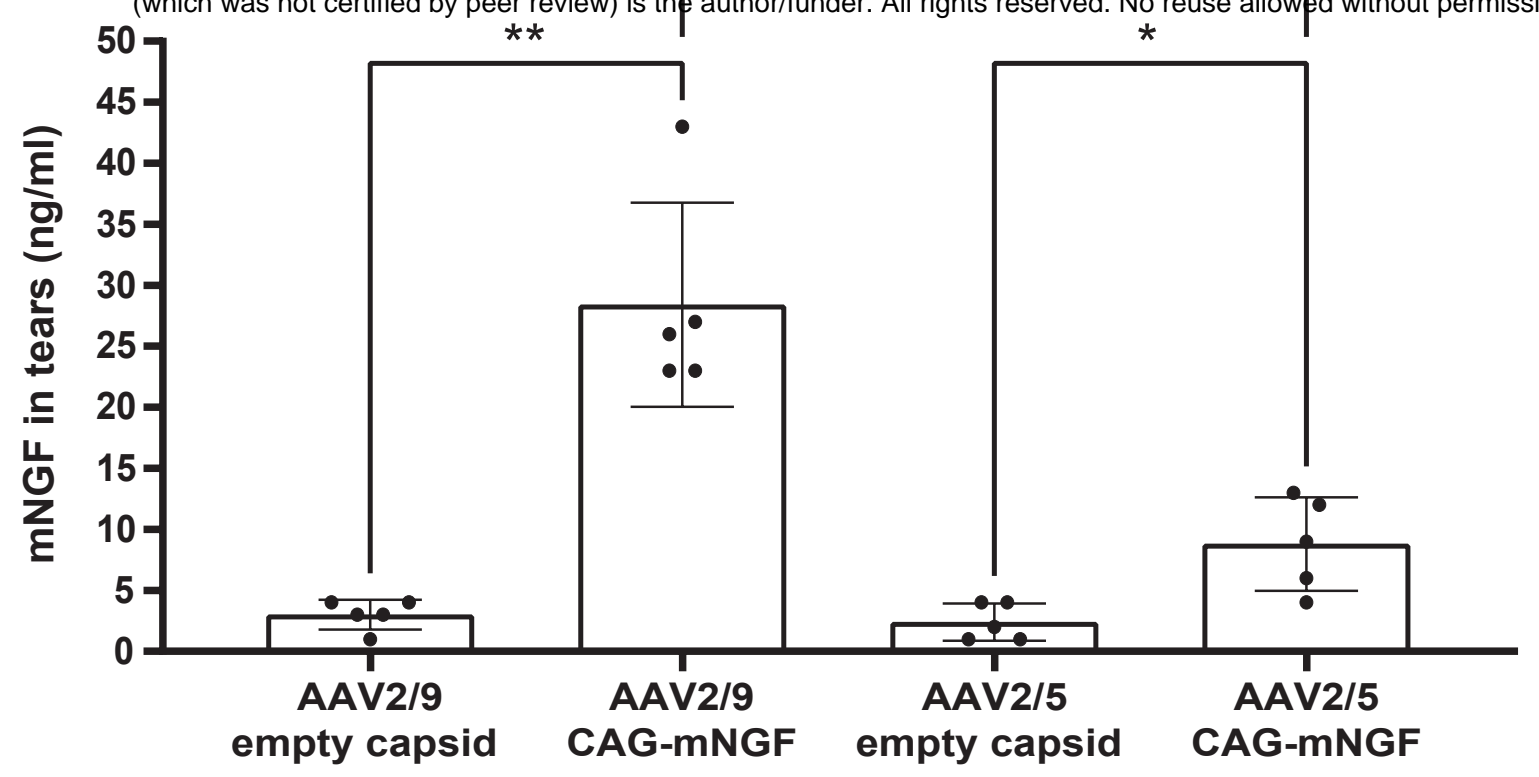




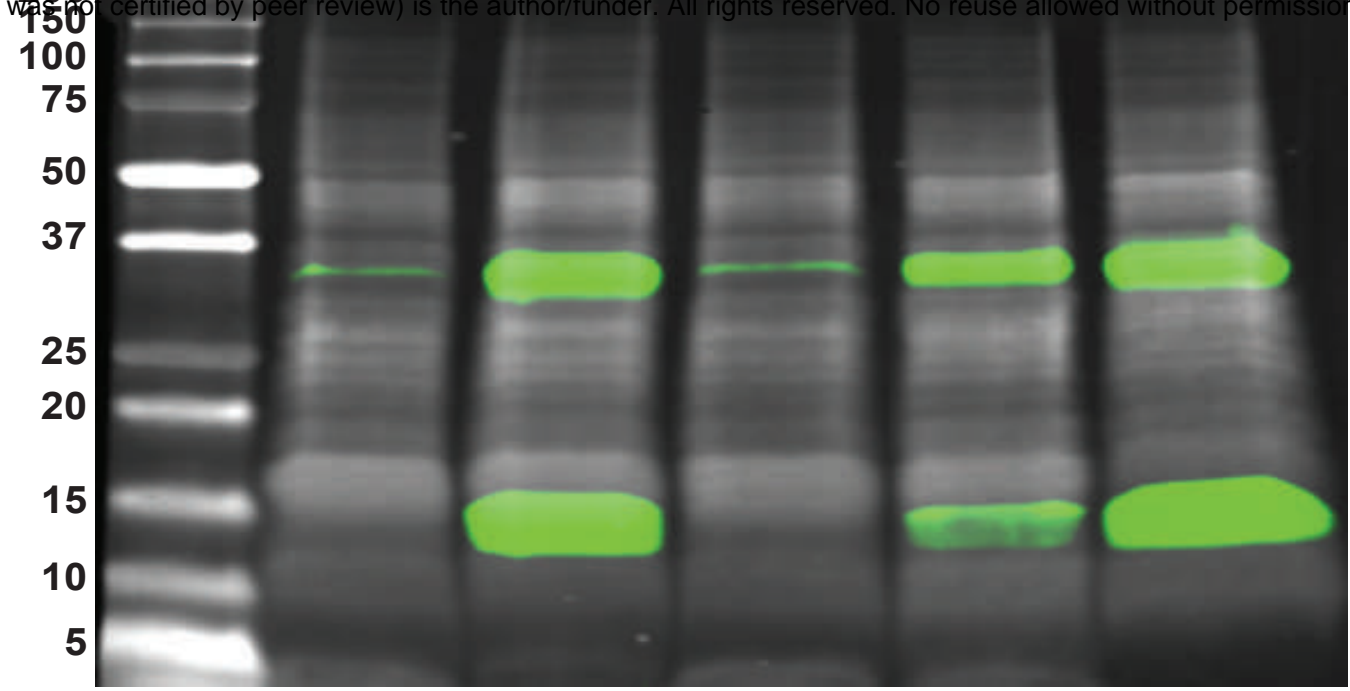

B

C
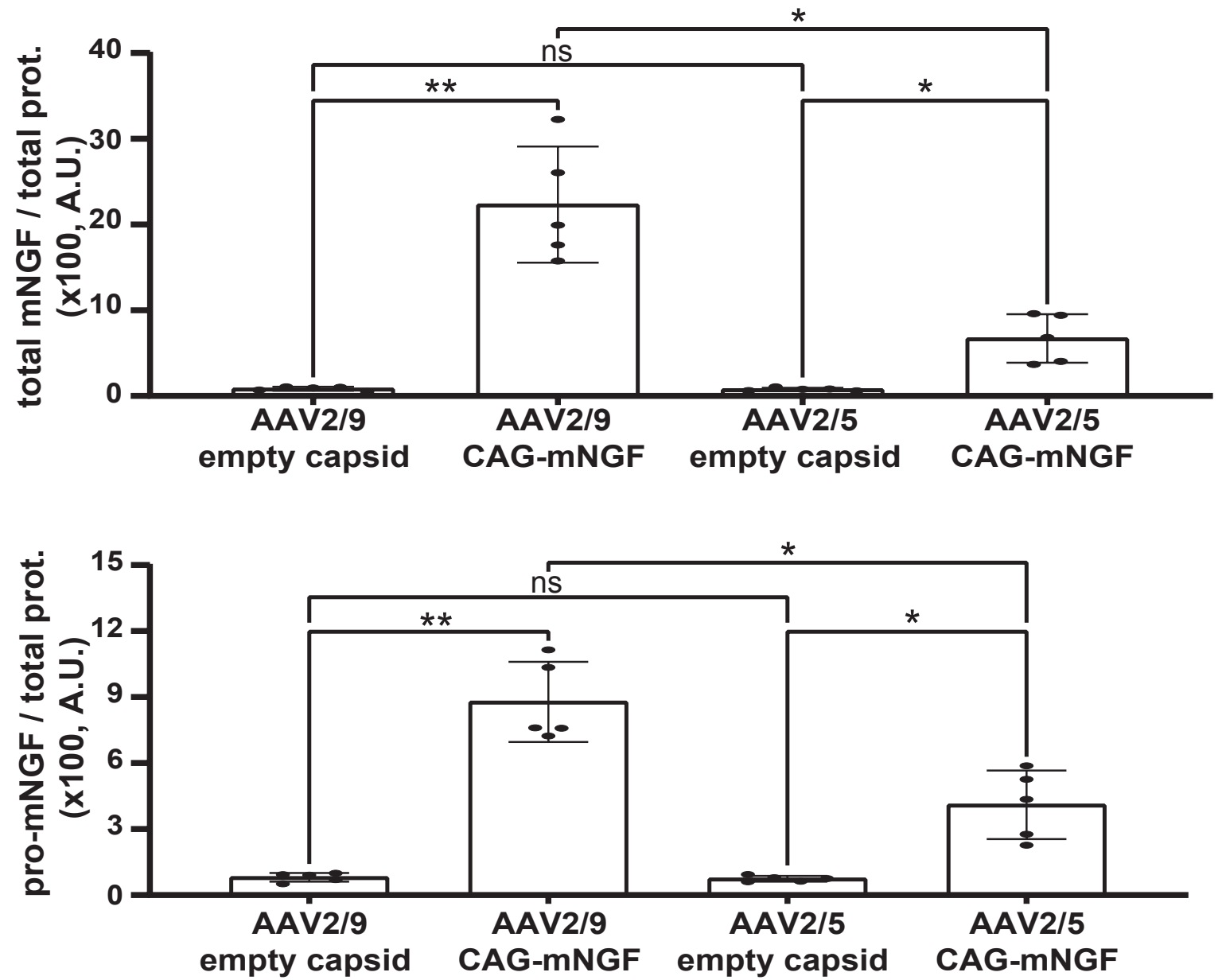

D

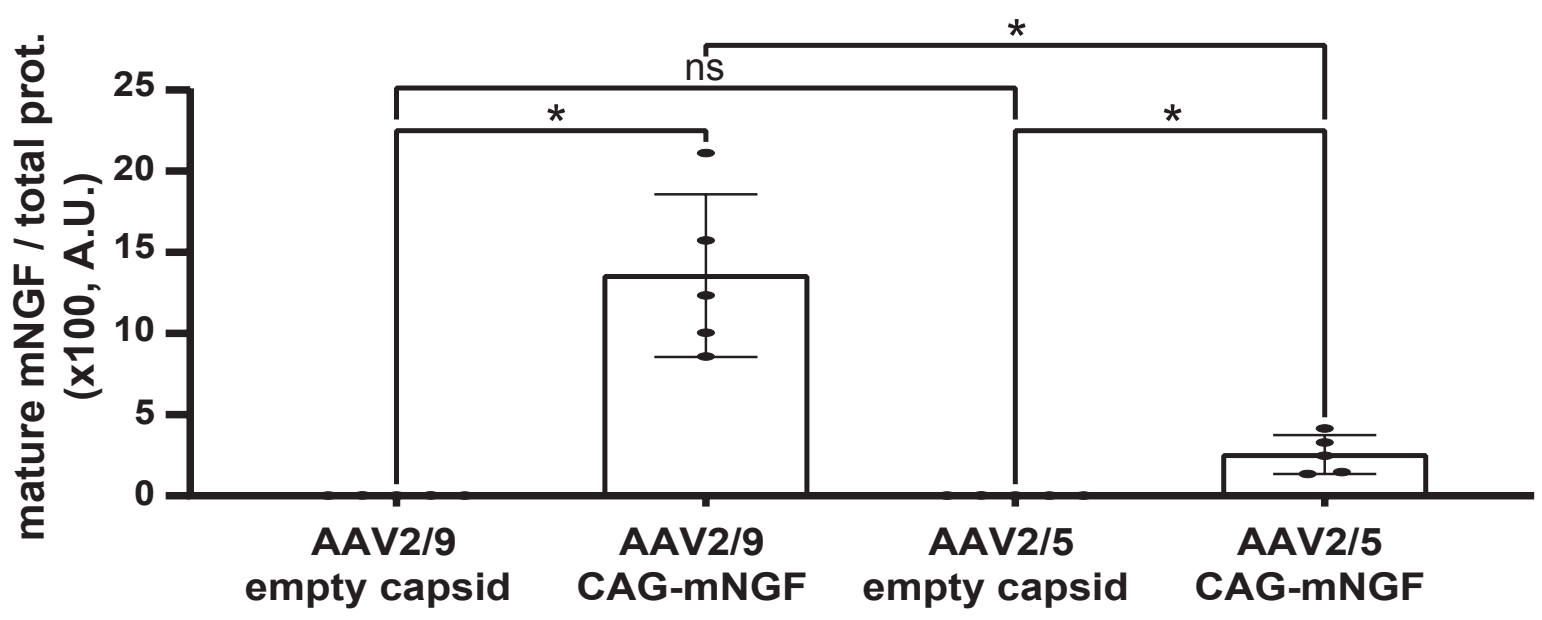


bioRXIV preprint doi: https://doi.org/10.1101/2022.02.16.480632; this version posted February 19, 2022. The copyright holder for this preprint (which was not certified by peer review) is the author/funder. All rights reserved. No reuse allowed without permission.

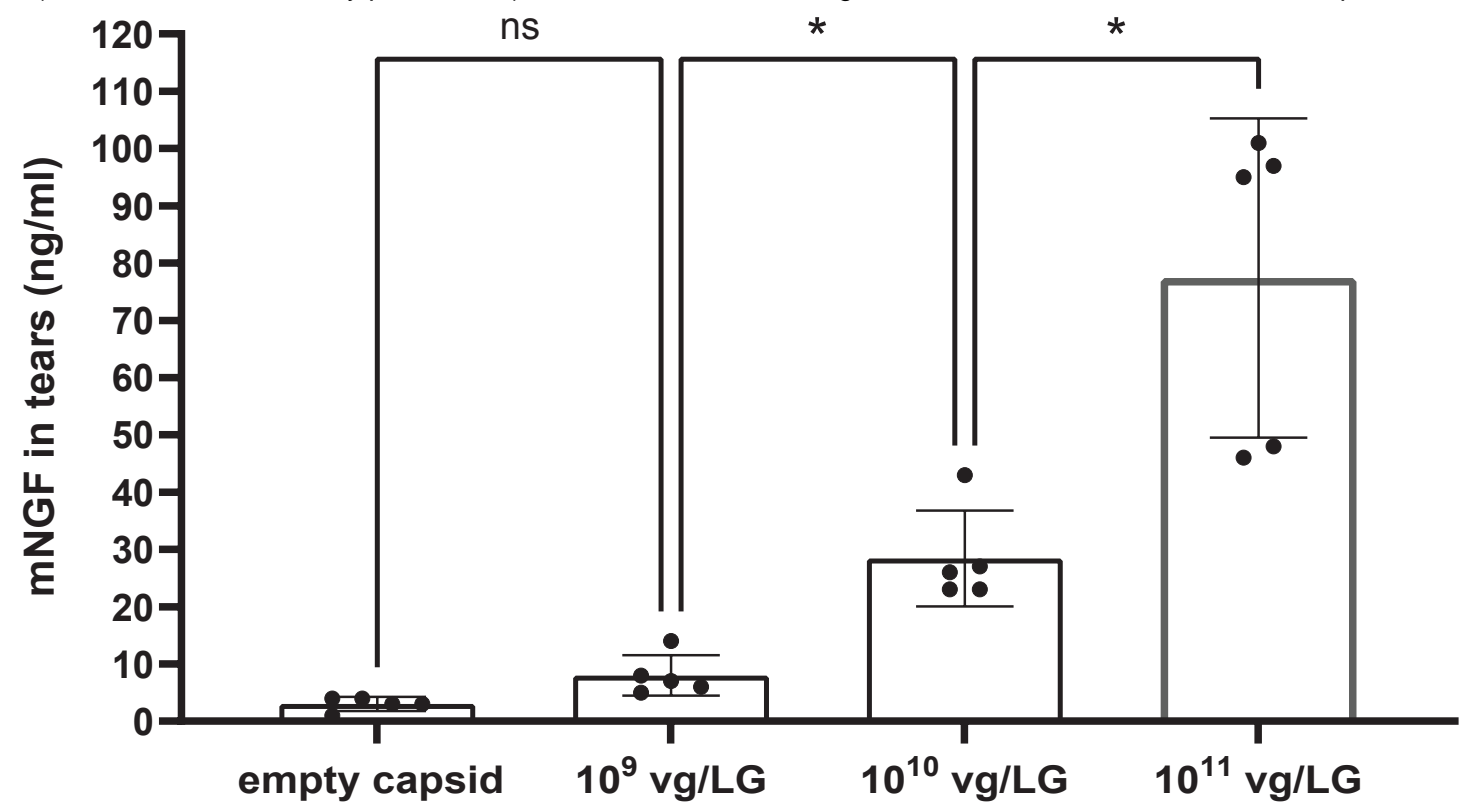

B

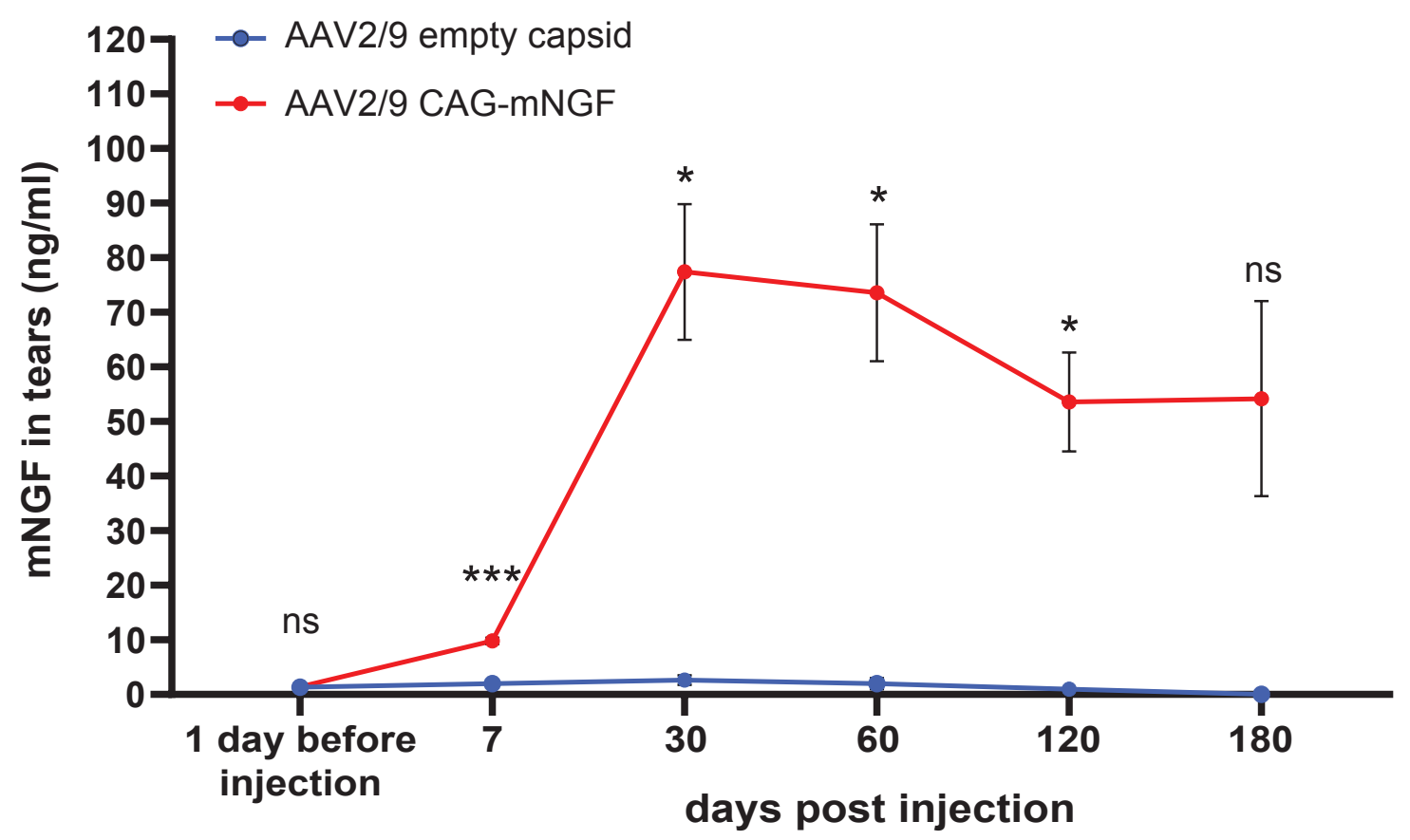


bioRxiv preprint doi: https://doi.org/10.1101/2022.02.16.480632; this version posted February 19, 2022. The copyright holder for this preprint (which was not certified by peer review) is the author/funder. All rights reserved. No reuse allowed without permission. A

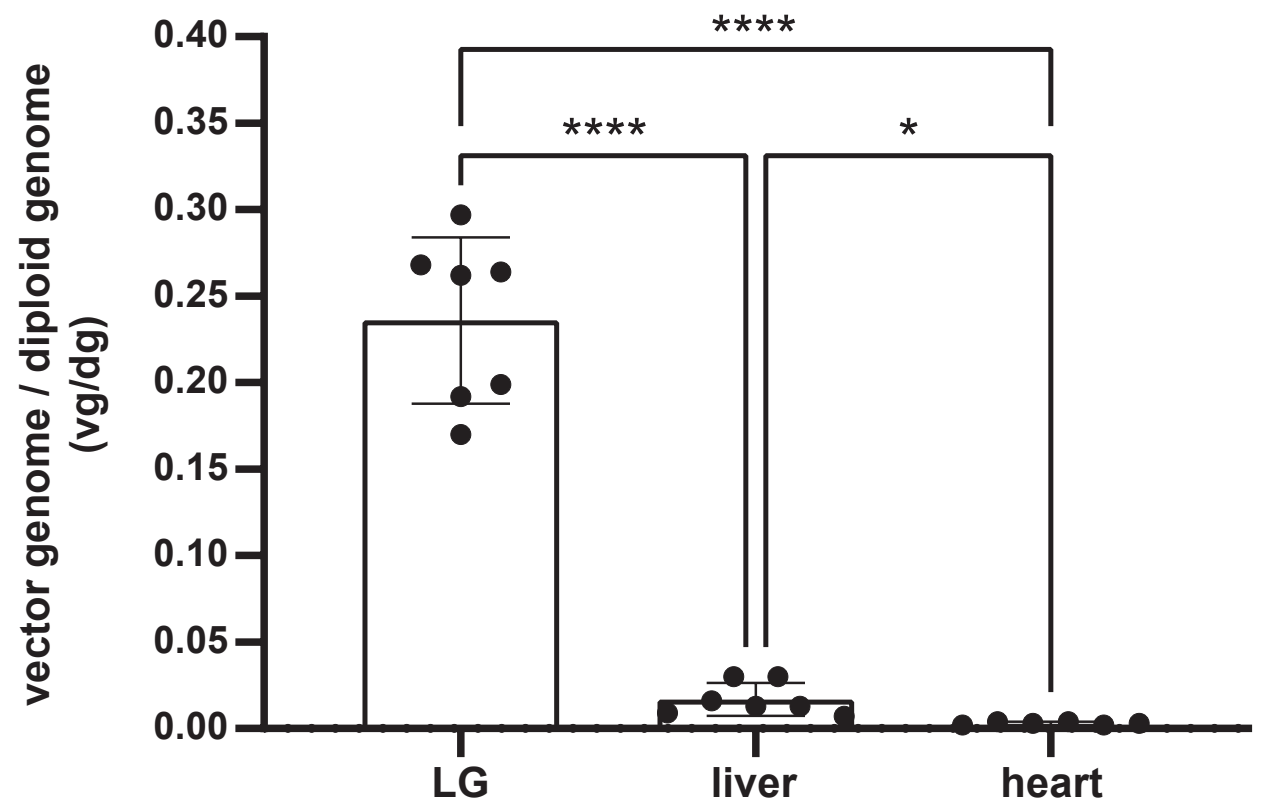

B

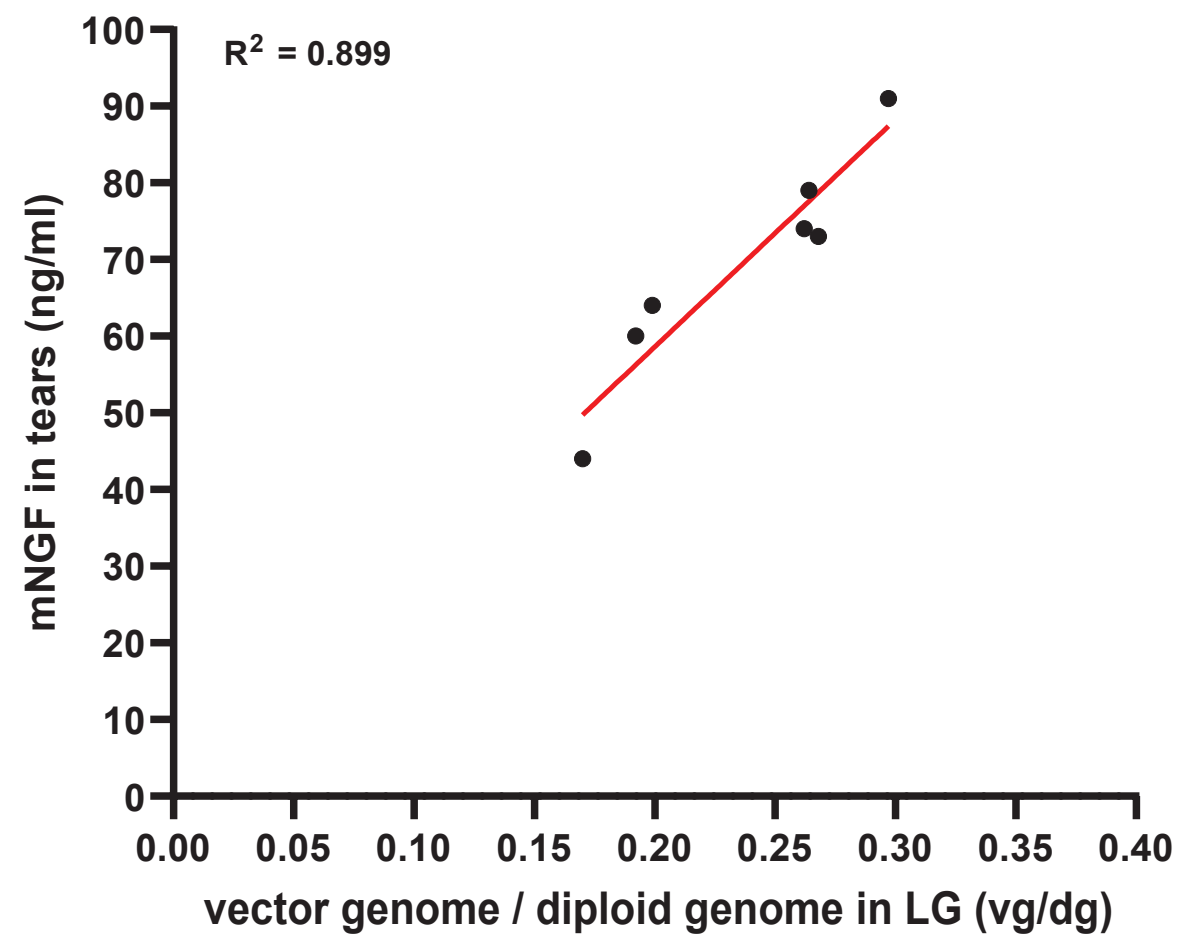


Figure 8

A bioRxiv preprint doi: https://doi.org/10.1101/2022.02.16.480632; this son posted February 19, 2022. The copyright holder for this preprint

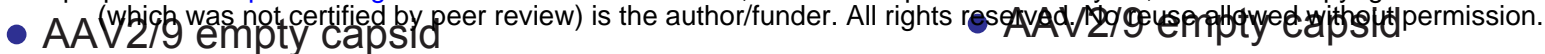

- AAV2/9 CAG-mNGF

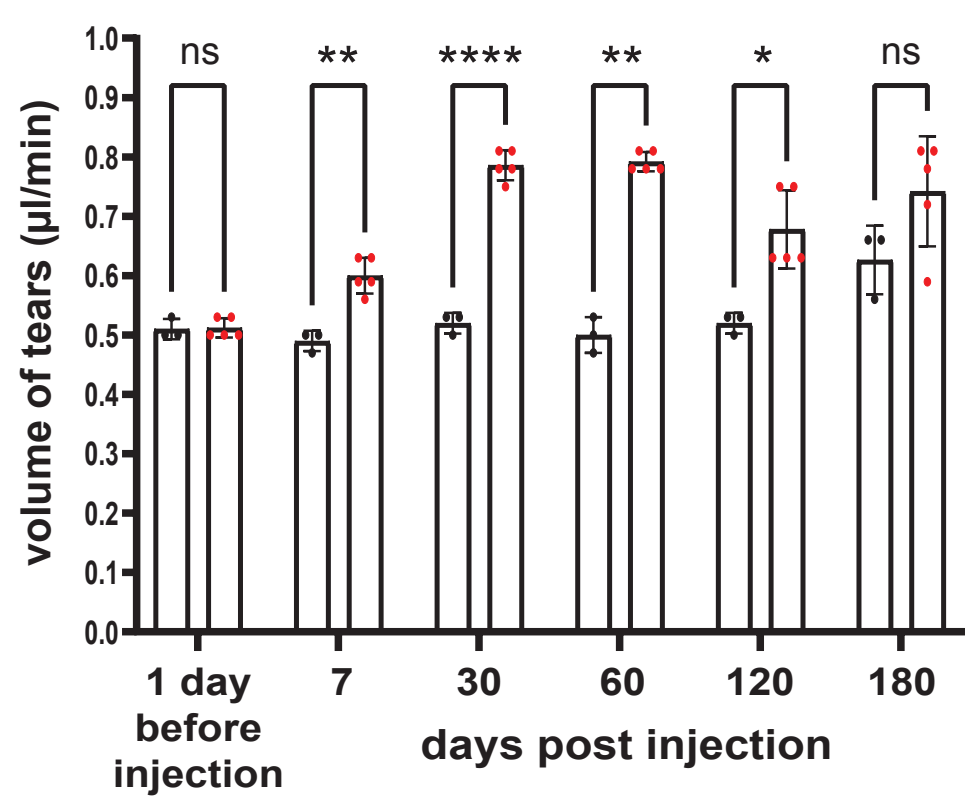

C
- AAV2/9 CAG-mNGF

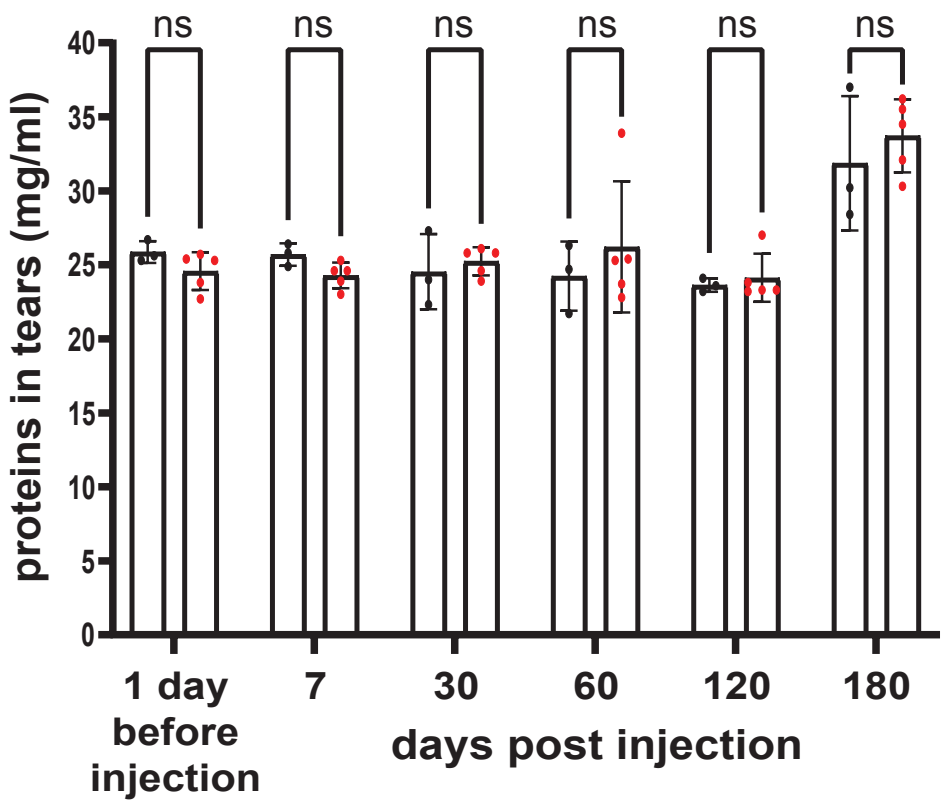

intact cornea abraded cornea
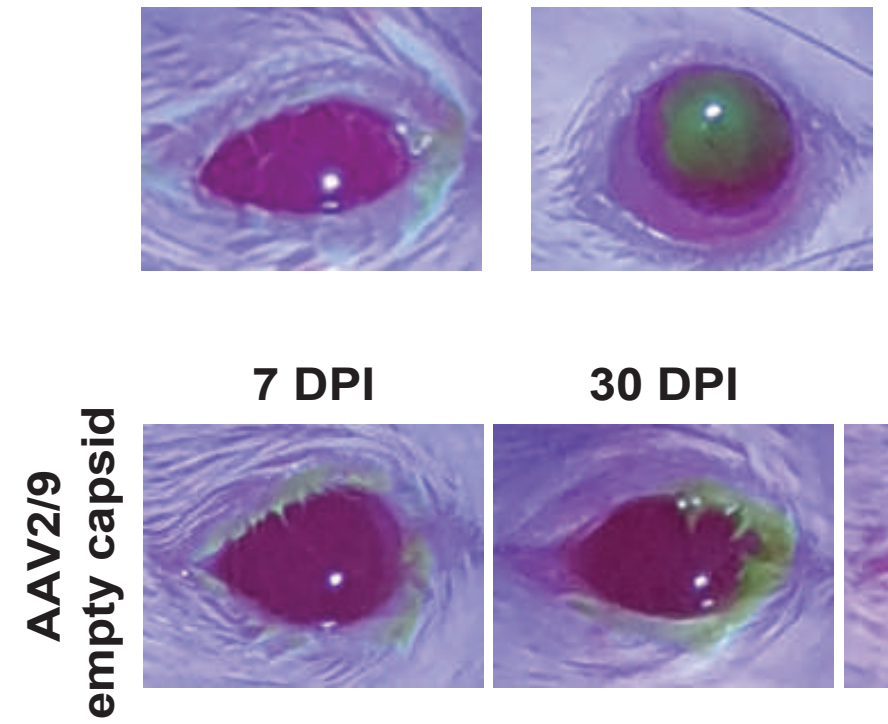

30 DPI

60 DPI

120 DPI

$180 \mathrm{DPI}$
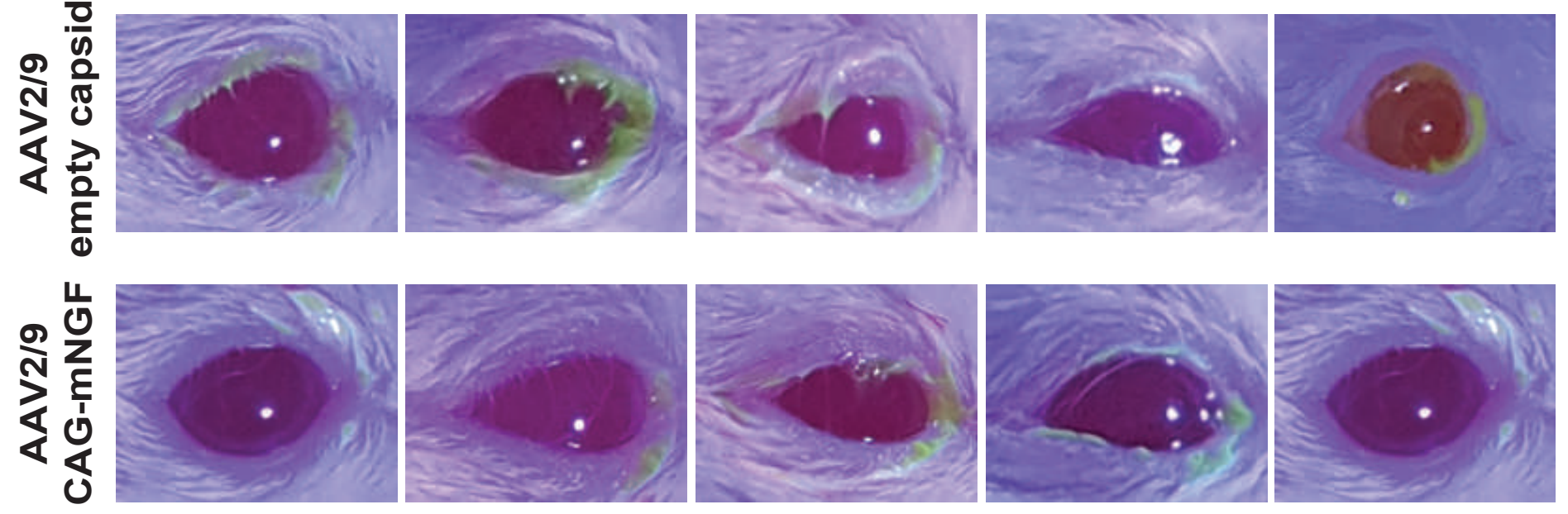


\section{Figure 9}

A bioRxiv preprint doi: https://doi.org/10.1101/2022.02.16.480632; this version posted February 19, 2022. The copyright holder for this preprint

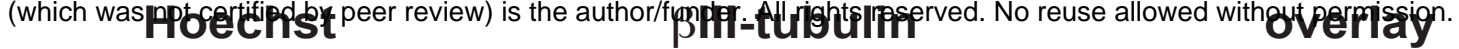
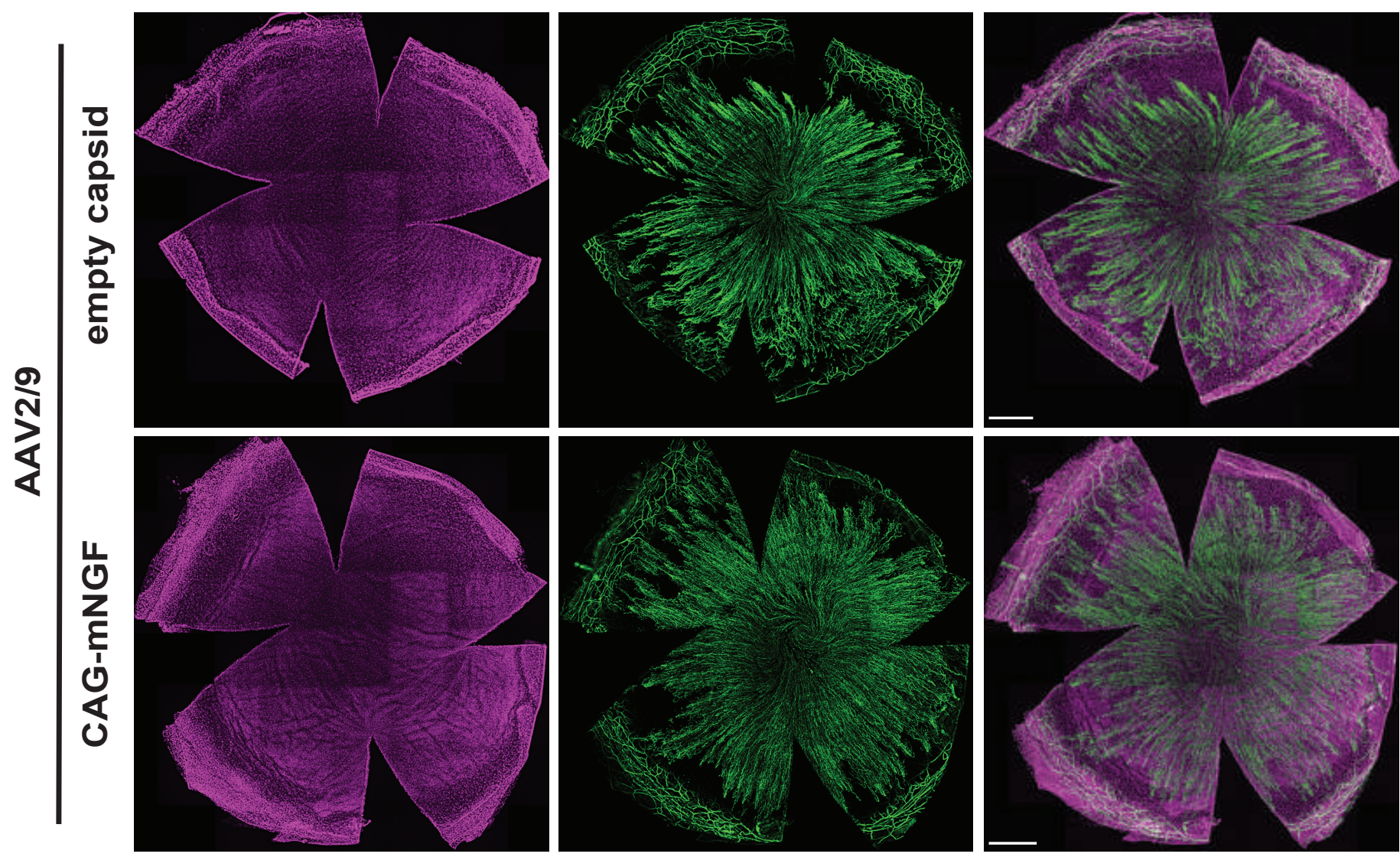

B

- AAV2/9 empty capsid

- AAV2/9 CAG-mNGF

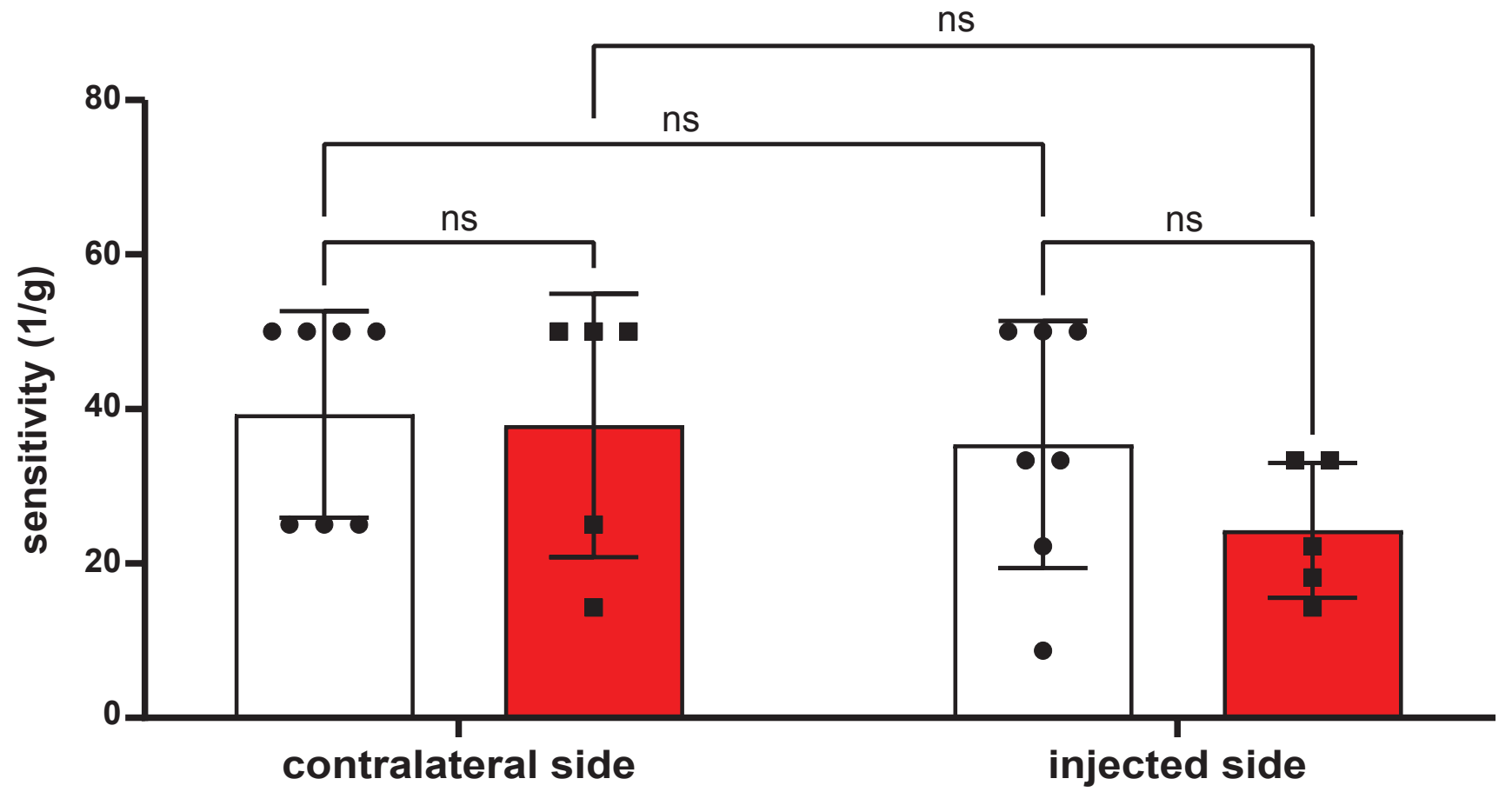

